Acta Crystallographica Section A

Foundations and

Advances

ISSN 2053-2733

Received 10 March 2014

Accepted 15 July 2014

(C) 2014 International Union of Crystallography

\section{An accurate parameterization for scattering factors, electron densities and electrostatic potentials for neutral atoms that obey all physical constraints}

\author{
I. Lobato* and D. Van Dyck \\ EMAT, University of Antwerp, Department of Physics, Groenenborgerlaan 171, B-2020, Antwerp, \\ Belgium. Correspondence e-mail: ivan.lobato@uantwerpen.be
}

\begin{abstract}
An efficient procedure and computer program are outlined for fitting numerical $\mathrm{X}$-ray and electron scattering factors with the correct inclusion of all physical constraints. The numerical electron scattering factors have been parameterized using five analytic non-relativistic hydrogen electron scattering factors as basis functions for 103 neutral atoms of the periodic table. The inclusion of the correct physical constraints in the electron scattering factor and its derived quantities allows the use of the new parameterization in different fields. In terms of quality of the fit, the proposed parameterization of the electron scattering factor is one order of magnitude better than the previous analytic fittings.
\end{abstract}

\section{Introduction}

In electron microscopy and electron diffraction the highenergy electrons interact with the atoms of the sample through their electrostatic Coulomb potential. This interaction is described by the high-energy Schrödinger equation and it can be solved numerically by different methods (Bethe, 1928; Howie \& Whelan, 1961; Cowley \& Moodie, 1957; Goodman \& Moodie, 1974; Ishizuka \& Uyeda, 1977; Van Dyck, 1979, 1980; Van Dyck \& Coene, 1984; Lobato \& Van Dyck, 2012). The simplest approach to describe the electrostatic specimen potential is by linear superposition of the spherically symmetric electrostatic potentials of each atom in the specimen. The atomic potential is related to the atomic charge distribution via Poisson's equation. It contains a contribution from the charge distribution of the electrons of the atom and a contribution from the nucleus, which can be considered as a point charge. In Fourier space, this then yields the atomic scattering factor given by the Mott-Bethe formula (Bethe, 1930; Mott, 1930). The electron charge distribution can be computed from the knowledge of the atomic wavefunction which can be obtained by numerically solving the Dirac equation under suitable approximations. The most reliable codes to calculate the atomic wavefunctions are the multiconfiguration Dirac-Fock (Desclaux, 1975; Grant et al., 1980; Dyall et al., 1989) and the relativistic Hartree-Fock codes (Grant, 1961, 1970; Coulthard, 1967). The Fourier transform of the electron charge distribution yields the X-ray scattering factor. By accounting for the contribution of the nucleus to the $\mathrm{X}$-ray scattering factor using the Mott-Bethe formula, one can then calculate the electron scattering factor.

Tabulated data are available for the X-ray and electron scattering factors for all the neutral atoms, which were computed using different methods and programs (Cromer \&
Waber, 1965; Doyle \& Turner, 1968; Fox et al., 1989; Rez et al., 1994; Kirkland, 1998). In the simulation programs of electron diffraction or high-resolution imaging, one can use the numerical values for the scattering factors and interpolate them to obtain the atomic potential or scattering factor at the required points. But in order to reduce the data and improve the numerical accuracy, one parameterizes the scattering factors using basic functions such as Gaussians and Lorentzians. Most parameterizations are obtained using a nonlinear least-square fitting with the tabulated data using the Levenberg-Marquardt algorithm (LMA) (Press et al., 2007). The LMA only finds a local minimum, which means that the result of fitting parameters depends sensitively on their initial assigned values. Originally one used only a sum of Gaussian functions (Smith \& Burge, 1962; Cromer \& Waber, 1965; Doyle \& Turner, 1968), which is sufficient for small spatial frequencies but cuts the scattering factors for large scattering angles. Weickenmeier \& Kohl (1991) used more complicated functions to fit the electron scattering factors to achieve a correct asymptotic behaviour for large angles. Kirkland (1998) used a combination of Lorentzians and Gaussians to fit the tabulated electron scattering data. The inclusion of the Lorentzians in this parameterization is needed to ensure the correct asymptotic behaviour for large angles. Although Kirkland used the LMA for the nonlinear minimization procedure, his fit was improved by trying several different starting points and keeping only the best one. Peng et al. (1996) used five Gaussians to fit the tabulated electron scattering data and performed a nonlinear least-square minimization by using simulated annealing (SA) optimization (Kirkpatrick et al., 1983). The drawback of all these parameterizations is that they are obtained by fitting with a discrete set of numerical values from which the asymptotic behaviour is then extrapolated. 
However, it has become increasingly clear that electron scattering and simulation programs should also include scattering at very large angles. Indeed, high-angle annular darkfield (HAADF) scanning transmission electron microscopy (STEM) calculations are done using a frozen-lattice model in which the atoms remain still and the scattering factors are not dampened by a Debye-Waller factor. The same argument holds for accurate computations of scattering into higherorder Laue zones (HOLZs). Moreover, from Rutherford's theory it is known that electrons can even backscatter if they hit the atomic nucleus. This means that reliable quantitative simulations for transmission electron microscopy (TEM) or STEM can only be done using an accurate parameterization for the electron scattering factors that are valid up to very large angles.

It is also a misconception that the X-ray scattering factors do not contribute to very large angles so that they can be parameterized adequately for the low spatial frequencies and that the asymptotic behaviour for very large spatial frequencies is given by the contribution of the positive nucleus which can be described analytically in the Mott-Bethe formula. But even the 1 state of the hydrogen atom, which can be calculated analytically, has an asymptotic contribution to large spatial frequencies that cannot be extrapolated from the current parameterizations.

The aim of this paper is to obtain an accurate parameterization for the elastic electron scattering factors from which the electron scattering factor and its derived quantities such as the X-ray scattering factor, the electron-density distribution and the atomic potential obey the correct physical constraint conditions, have the correct asymptotic behaviour and can be calculated analytically.

This paper is organized as follows. In $\S 2.1$, we discuss the choice of a set of basis functions for the expansion of the electron scattering factor. The physical constraint conditions and their proper inclusion in the fitting procedure are presented in $\S \S 2.2$ and 2.3 , respectively. $\S 2.4$ describes the nonlinear minimization process used for the parameterization. Results and discussion are presented in $\$ 3$, and finally the main conclusions are given.

\section{Parameterization of the elastic electron scattering factors}

\subsection{Basis functions for the parameterization of the elastic electron scattering factors}

We want to find a natural set of basis functions in which the elastic electron scattering factors can be properly expanded. To accomplish this goal, let us start with some general definitions (Kirkland, 1998).

The X-ray scattering factor for a spherically symmetric electron charge distribution $\rho(r)$ is defined as

$$
f_{\mathrm{x}}(g)=\int_{0}^{\infty} 4 \pi r^{2} \rho(r) \frac{\sin (2 \pi g r)}{2 \pi g r} \mathrm{~d} r,
$$

where $r$ and $g$ are the magnitude of the three-dimensional vectors $\mathbf{r}$ and $\mathbf{g}$, respectively. $f_{\mathrm{x}}(g)$ is a dimensionless quantity corresponding to the distribution of electrons. For neutral atoms $f_{\mathrm{x}}(0)=Z$, where $Z$ is the atomic number.

By definition the electron scattering factor is related to the atomic potential $V(\mathbf{r})$ by means of its three-dimensional Fourier transform:

$$
f_{\mathrm{e}}(\mathbf{g})=\kappa \int V(\mathbf{r}) \exp (-2 \pi \mathbf{g} \cdot \mathbf{r}) \mathrm{d} \mathbf{r},
$$

where $\kappa=\left(4 \pi \varepsilon_{0}\right) /\left(2 \pi a_{0} e\right), e$ is the electron charge, $\varepsilon_{0}$ the vacuum permittivity and $a_{0}$ the Bohr radius. $f_{\mathrm{e}}(g)$ is in units of $\AA$. For the case where the atom is spherically symmetric this reduces to

$$
f_{\mathrm{e}}(g)=\kappa \int_{0}^{\infty} 4 \pi r^{2} V(r) \frac{\sin (2 \pi g r)}{2 \pi g r} \mathrm{~d} r .
$$

For an atom with atomic number $Z$, the electron scattering factor is related to the X-ray scattering factor $f_{\mathrm{x}}(g)$ via the Mott-Bethe formula (Bethe, 1930; Mott, 1930):

$$
f_{\mathrm{e}}(g)=\frac{1}{2 \pi^{2} a_{0}}\left[\frac{Z-f_{\mathrm{x}}(g)}{g^{2}}\right] \text {. }
$$

The Mott-Bethe formula is equivalent to the solution of Poisson's equation in reciprocal space yielding the potential distribution from the charge distribution. Equation (4) presents a singularity at $g=0$ and it must be replaced by the following expression given by Ibers (1958):

$$
\begin{gathered}
f_{\mathrm{e}}(0)=\frac{4 \pi^{2} m_{0} e^{2}}{3 h^{2}} Z\left\langle r^{2}\right\rangle, \\
\left\langle r^{2}\right\rangle=\frac{\int_{0}^{\infty} r^{2}\left[4 \pi r^{2} \rho(r)\right] \mathrm{d} r}{\int_{0}^{\infty}\left[4 \pi r^{2} \rho(r)\right] \mathrm{d} r},
\end{gathered}
$$

where $m_{0}$ is the rest mass of the electron, $h$ is Planck's constant and $\left\langle r^{2}\right\rangle$ is the mean-square distance of the electron distribution in the atom. Note that neutral atoms also satisfy

$$
Z=\int_{0}^{\infty} 4 \pi r^{2} \rho(r) \mathrm{d} r
$$

The hydrogen atom is the only one for which the (nonrelativistic) electron charge density $\rho^{\mathrm{H}}(r)$, the X-ray scattering factor $f_{\mathrm{x}}^{\mathrm{H}}(g)$, the electron scattering factor $f_{\mathrm{e}}^{\mathrm{H}}(g)$, the atomic potential $V^{\mathrm{H}}(r)$ and the projected atomic potential $V_{z}^{\mathrm{H}}(R)$ can be calculated analytically:

$$
\begin{gathered}
\rho^{\mathrm{H}}(r)=\frac{1}{\pi a_{0}^{3}} \exp \left(-2 \pi r / a_{0}\right), \\
f_{\mathrm{x}}^{\mathrm{H}}(g)=\frac{1}{\left(1+\pi^{2} a_{0}^{2} g^{2}\right)^{2}}, \\
f_{\mathrm{e}}^{\mathrm{H}}(g)=\frac{a_{0}}{2}\left[\frac{1}{1+\pi^{2} a_{0}^{2} g^{2}}+\frac{1}{\left(1+\pi^{2} a_{0}^{2} g^{2}\right)^{2}}\right] \\
V^{\mathrm{H}}(r)=\frac{e}{4 \pi \varepsilon_{0}}\left(\frac{\exp \left(-2 r / a_{0}\right)}{r}+\frac{\exp \left(-2 r / a_{0}\right)}{a_{0}}\right),
\end{gathered}
$$




$$
V_{z}^{\mathrm{H}}(R)=\frac{e}{4 \pi \varepsilon_{0}}\left[2 K_{0}\left(\frac{2 R}{a_{0}}\right)+\frac{2 R}{a_{0}} K_{1}\left(\frac{2 R}{a_{0}}\right)\right],
$$

where $R$ is the magnitude of the two-dimensional vector. $K_{0}(x)$ and $K_{1}(x)$ are the modified Bessel functions of the second kind of order zero and one, respectively.

Although $\rho(r)$ was calculated by numerically solving the Dirac equation, we use the non-relativistic electron scattering factor of the hydrogen as basis function to parameterize the electron scattering factor for all the neutral atoms. We find out that it is better to parameterize $f_{\mathrm{e}}(g)$ for all the atoms by taking as basis function the total electron scattering factor of the hydrogen atom instead of independent sums of Lorentzians and squared Lorentzians. The choice of this basis function for the expansion of the electron scattering factors combined with the Riemann-Lebesgue lemma guarantees that the electron density will be an integrable function with no singularity and discontinuity. Hence, we express the electron scattering factor in the following form:

$$
f_{\mathrm{e}}(g)=\sum_{i=1}^{n_{t}} a_{i}\left[\frac{1}{\left(1+b_{i} g^{2}\right)}+\frac{1}{\left(1+b_{i} g^{2}\right)^{2}}\right]=\sum_{i=1}^{n_{t}} a_{i} \frac{\left(2+b_{i} g^{2}\right)}{\left(1+b_{i} g^{2}\right)^{2}}
$$

where $n_{t}$ is the number of non-relativistic electron scattering factors of the hydrogen form. This parameterization is expected to fit better for low atomic numbers where the relativistic effects are not prominent.

This parameterized form of the electron scattering factor shows the correct asymptotic behaviour according to the Mott-Bethe formula (4), which implies that the electron scattering factor has to decay proportional to $1 / g^{2}$ for large angles.

Now, the X-ray scattering factor can be obtained analytically using the Mott-Bethe formula (4):

$$
f_{\mathrm{x}}(g)=Z-2 \pi^{2} a_{0} g^{2} \sum_{i=1}^{n_{t}} a_{i} \frac{\left(2+b_{i} g^{2}\right)}{\left(1+b_{i} g^{2}\right)^{2}}
$$

and the atomic potential can be deduced by the inverse threedimensional Fourier transform of the electron scattering factor [equation (13)]:

$$
V(r)=\frac{1}{\kappa} \sum_{i=1}^{n_{t}} \frac{\pi^{2} a_{i}}{b_{i}^{3 / 2}}\left(\frac{b_{i}^{1 / 2}}{\pi r}+1\right) \exp \left(\frac{-2 \pi r}{b_{i}^{1 / 2}}\right)
$$

This equation expresses the atomic potential as a sum of Yukawa and exponential functions. For a point-like nucleus charge the Yukawa terms give the correct asymptotic behaviour at small radial distance.

The projected potential can also be obtained analytically by integration of the atomic potential along the $z$ axis:

$$
V(R)=\frac{1}{\kappa} \sum_{i=1}^{n_{t}} \frac{2 \pi^{2} a_{i}}{b_{i}^{3 / 2}}\left[K_{0}\left(\frac{2 \pi R}{b_{i}^{1 / 2}}\right)+R K_{1}\left(\frac{2 \pi R}{b_{i}^{1 / 2}}\right)\right] .
$$

\subsection{Constraint conditions}

The first constraint arises due to the fact that $f_{\mathrm{e}}(0)$ represents a physical and measurable quantity which is proportional to the mean-square distance of the electrons in the atom [equation (5)]. Therefore, we impose the constraint that the parameterized form of the electron scattering factor has to reproduce the exact tabulated value of $f_{\mathrm{e}}(0)$ :

$$
\sum_{i=1}^{n_{t}} 2 a_{i}=f_{\mathrm{e}}(0)
$$

The second constraint stems from applying the RiemannLebesgue lemma to the electron density, which requires that the X-ray scattering factor has to decay to zero when $g$ approaches infinity:

$$
\lim _{g \rightarrow \infty} f_{\mathrm{x}}(g)=0
$$

which, for the derived parameterization of the X-ray scattering factor in equation (14), gives the following constraint for the coefficients:

$$
\sum_{i=1}^{n_{t}} \frac{a_{i}}{b_{i}}=\frac{Z}{2 \pi^{2} a_{0}}
$$

Using this constraint, the X-ray scattering factor in equation (14) can be rewritten in a more compact form as

$$
f_{\mathrm{x}}(g)=2 \pi^{2} a_{0} \sum_{i=1}^{n_{t}} \frac{a_{i}}{b_{i}\left(1+b_{i} g^{2}\right)^{2}} .
$$

The electron charge density $\rho(r)$ can be obtained analytically by taking the inverse three-dimensional Fourier transform of equation (20), that gives us

$$
\rho(r)=2 \pi^{2} a_{0} \sum_{i=1}^{n_{t}} \frac{\pi^{2} a_{i}}{b_{i}^{5 / 2}} \exp \left(\frac{-2 \pi r}{b_{i}{ }^{1 / 2}}\right) .
$$

This equation expresses $\rho(r)$ as a sum of exponential functions, which is consistent with the numerical observation that the atomic electron densities are nearly piecewise exponential (Sperber, 1971; Weinstein et al., 1975; Wang \& Parr, 1977).

It is known that for point-like nuclei the non-relativistic electron density fulfils the cusp condition, which was derived by Steiner as a corollary to Kato's theorem (Steiner, 1963; Kato, 1957). However, the relativistic electron density does not exhibit a cusp at the position of atomic nuclei if a finitesized nuclear charge distribution is assumed. Instead a constant electron density is found at the position of an atomic nucleus (Mastalerz et al., 2010). Owing to the fact that the electron scattering factors are derived from the X-ray scattering factors using the Mott-Bethe formula (Bethe, 1930; Mott, 1930), which is derived for point-like nuclei, we can still use Kato's cusp condition as an exact constraint. Kato's cusp condition states that the electron density of the ground state has a cusp at the position of the nucleus, where it satisfies

$$
\left.\frac{\partial \rho(r)}{\partial r}\right|_{r=0}=\frac{-2 Z}{a_{0}} \rho(0) .
$$


After applying this condition to the derived electron charge density in equation (21) we arrive at

$$
\sum_{i=1}^{n_{t}} \frac{a_{i}}{b_{i}^{5 / 2}}\left(\frac{\pi}{b_{i}^{1 / 2}}-\frac{Z}{a_{0}}\right)=0 .
$$

To avoid divergences in the electron charge density and to guarantee that it is an integrable function, the square root of the nonlinear parameters $b_{i}$ has to be positive. This give zero as a lower bound for the $b_{i}$ coefficients. On the other hand, there is no restriction for the linear parameters $a_{i}$ as they may take positive and negative values. The parameterized electron scattering factor for a neutral atom has to guarantee that the spherically averaged $\rho(r)$ and $V(r)$ are positive everywhere and decrease monotonically along the radial direction from the nucleus to zero at infinity (Sperber, 1971; Weinstein et al., 1975; Wang \& Parr, 1977; Sen \& Politzer, 1989).

\subsection{Cost function and inclusion of constraints}

We perform a nonlinear least-square fit using simulated annealing for the numerical tabulation of the X-ray scattering factors $f_{\mathrm{x} j}$ and electron scattering factors $f_{\mathrm{e} j}$ at the angle $g_{j}$ to the parameterized form of $f_{\mathrm{e}}\left(g_{j}\right)$ in equation (13). Our cost function is based on a modified version of the combined reduce merit function used by Kirkland (1998),

$$
\chi^{2}=\frac{1}{2 n_{g}} \sum_{i=1}^{n_{g}}\left(\left[\frac{f_{\mathrm{e} i}-f_{\mathrm{e}}\left(g_{i}\right)}{f_{\mathrm{e} 0}}\right]^{2}+\left\{\varpi_{i}\left[\frac{f_{\mathrm{xi}}-f_{\mathrm{x}}\left(g_{i}\right)}{f_{\mathrm{x} 0}}\right]\right\}^{2}\right),
$$

where $\varpi_{i}=\left(f_{\mathrm{e} i} / f_{\mathrm{e} 0}\right) /\left(f_{\mathrm{x} i} / f_{\mathrm{x} 0}\right)$ is used to give equal weights to the X-ray and electron scattering factors. The choice of these weight factors gives slight preference for matching the electron scattering factors than the X-ray scattering factors. The latter equation can be expressed in reduced form by means of the inverse Mott-Bethe formula:

$$
\chi^{2}=\sum_{i=1}^{n_{g}}\left\{w_{i}\left[f_{\mathrm{e} i}-f_{\mathrm{e}}\left(g_{i}\right)\right]\right\}^{2},
$$

where

$$
w_{i}=\left[\frac{1+\left(\frac{Z-f_{\mathrm{x} i}}{f_{\mathrm{x} i}}\right)^{2}}{2 n_{\mathrm{g}} f_{\mathrm{e} 0}^{2}}\right]^{1 / 2} .
$$

Let us define $n_{b}$ to be the number of exact constraint conditions to be imposed on the parameterization and $n_{b c}=$ $n_{t}-n_{b}$. If we split the linear fitting parameters in two column vectors as $A=\left\{a_{i}\right\}, i=1, \ldots, n_{b}$ and $B=\left\{a_{i}\right\}$, $i=n_{b}+1, \ldots, n_{t}$, then the constraint conditions can be expressed in a matrix equation,

$$
M A=N B+C,
$$

where $M=\left\{M_{i j}\right\}_{n_{b} \times n_{b}}, N=\left\{N_{i j}\right\}_{n_{b} \times n_{b c}}$ and $C=\left\{C_{i}\right\}_{n_{b} \times 1}$ are determined by the exact boundary conditions given in equations (17), (19) and (23).

For a given set of $n_{g}$ numerical electron scattering factors $f_{\mathrm{e} i}, i=1, \ldots, n_{g}, n_{g}$ linear algebraic equations can be written down for $n_{t}$ linear parameters,

$$
w_{i} f_{\mathrm{e} i}=w_{i}\left[\sum_{j=1}^{n_{t}} a_{j} \frac{\left(2+b_{j} g_{i}^{2}\right)}{\left(1+b_{j} g_{i}^{2}\right)^{2}}\right] .
$$

If we split the last set of linear equations in two groups as

$$
w_{i} f_{\mathrm{e} i}=\sum_{j=1}^{n_{b}} \frac{w_{i}\left(2+b_{j} g_{i}^{2}\right)}{\left(1+b_{j} g_{i}^{2}\right)^{2}} a_{j}+\sum_{j=n_{b}+1}^{n_{t}} \frac{w_{i}\left(2+b_{j} g_{i}^{2}\right)}{\left(1+b_{j} g_{i}^{2}\right)^{2}} a_{j},
$$

then it can be expressed in matrix form as

$$
w_{i} f_{\mathrm{e} i}=I A+J B,
$$

where $I=\left\{I_{i j}\right\}_{n_{g} \times n_{b}}$ and $J=\left\{J_{i j}\right\}_{n_{g} \times n_{b c}}$ are matrices determined from equation (29).

From equation (27) $A$ can be expressed as

$$
A=M^{-1}(N B+C) \text {. }
$$

Substituting $A$ into equation (30) and after rearranging terms we obtain

$$
F=R B
$$

where

$$
\begin{gathered}
F=w_{i} f_{\mathrm{e} i}-S C, \\
R=S N+J
\end{gathered}
$$

and $S$ is obtained by solving the following matrix equation:

$$
M^{T} S^{T}=I^{T} \text {. }
$$

In the present case $n_{g}>n_{b c}$, then equation (32) is overdetermined. To find an approximate solution that satisfies all the equations simultaneously, the following linear least-square method is used:

$$
\operatorname{minimize}_{B}|F-R B|^{2} \text {. }
$$

Its solution can be written by the normal equations

$$
B=\left(R^{T} R\right)^{-1} R^{T} F .
$$

In order to solve the linear least-square problem for a given set of coefficients $b_{i}$ and achieve good numerical accuracy, we use a singular value decomposition (SVD) of the matrix $R$. Once $B$ is obtained, the $A$ vector is calculated by means of equation (31), and therefore all the linear coefficients $a_{i}$.

\subsection{Nonlinear least-square fitting}

The minimization process of the cost function $\chi^{2}$ is performed using the SA technique (Kirkpatrick et al., 1983) and the linear least-square method for solving the linear algebraic equations. The SA method statistically guarantees to find an optimal solution (in an infinite time) and it processes cost functions with a high degree of nonlinearity, arbitrary boundary conditions and constraints imposed on the cost function (Ingber, 1993). In this work, we have used the modified SA with adaptive neighbourhood based on the Corona method (Corona et al., 1987).

For a given set of $b=\left\{b_{i}\right\}$ nonlinear parameters, a new set of $b^{\prime}=\left\{b_{i}^{\prime}\right\}$ nonlinear parameters is generated by the following equation: 
Table 1

The fitting coefficients for the elastic electron scattering factors.

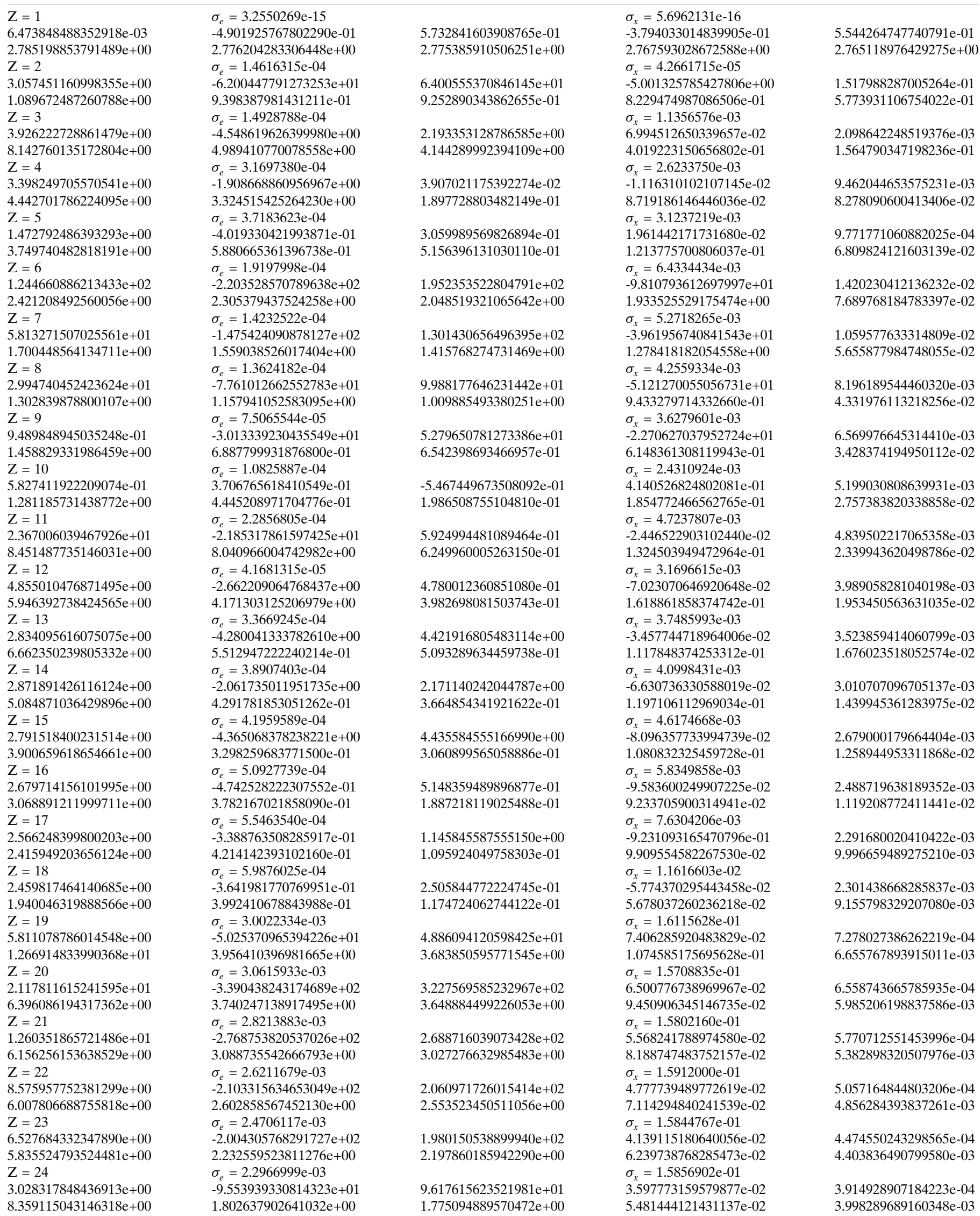


Table 1 (continued)

\begin{tabular}{|c|c|c|c|c|}
\hline$Z=25$ & $\sigma_{e}=2.2029500 \mathrm{e}-03$ & & $\sigma_{x}=1.5626624 \mathrm{e}-01$ & \\
\hline $4.374175506331227 \mathrm{e}+00$ & $-1.609255109187795 e+02$ & $1.602733080603223 e+02$ & $3.123038610491624 \mathrm{e}-02$ & $3.469660210209380 \mathrm{e}-04$ \\
\hline $5.510317055049282 \mathrm{e}+00$ & $1.687982164024339 \mathrm{e}+00$ & $1.666140477777024 \mathrm{e}+00$ & $4.833703903212770 \mathrm{e}-02$ & $3.647469600530682 \mathrm{e}-03$ \\
\hline$Z=26$ & $\sigma_{e}=2.0916073 \mathrm{e}-03$ & & $\sigma_{x}=1.5453291 \mathrm{e}-01$ & \\
\hline $3.798100908368596 \mathrm{e}+00$ & $-9.168935493816872 \mathrm{e}+01$ & $9.144542521554298 \mathrm{e}+01$ & $2.727543440276047 \mathrm{e}-02$ & $3.033798543837710 \mathrm{e}-04$ \\
\hline $5.317126458994331 \mathrm{e}+00$ & $1.497130948847481 \mathrm{e}+00$ & $1.468092418044105 \mathrm{e}+00$ & $4.272478501289549 \mathrm{e}-02$ & $3.327918552318741 \mathrm{e}-03$ \\
\hline$Z=27$ & $\sigma_{e}=2.0008539 \mathrm{e}-03$ & & $\sigma_{x}=1.5124769 \mathrm{e}-01$ & \\
\hline $3.330378744675443 \mathrm{e}+00$ & $-7.700175964729671 e+01$ & $7.707252217905169 \mathrm{e}+01$ & $2.399046690405699 \mathrm{e}-02$ & $2.682566655239420 \mathrm{e}-04$ \\
\hline $5.181359645795432 \mathrm{e}+00$ & $1.329151222651394 \mathrm{e}+00$ & $1.302849289632289 \mathrm{e}+00$ & $3.806454868263710 \mathrm{e}-02$ & $3.050100079915708 \mathrm{e}-03$ \\
\hline $\mathrm{Z}=28$ & $\sigma_{e}=1.9044267 \mathrm{e}-03$ & & $\sigma_{x}=1.4902251 \mathrm{e}-01$ & \\
\hline $2.969080787252864 \mathrm{e}+00$ & $-7.574770691290402 \mathrm{e}+01$ & $7.603982876253073 \mathrm{e}+01$ & $2.101621136929670 \mathrm{e}-02$ & $2.311517511351530 \mathrm{e}-04$ \\
\hline $5.041809491093800 \mathrm{e}+00$ & $1.182755079216293 \mathrm{e}+00$ & $1.162165458466299 \mathrm{e}+00$ & $3.374790878830353 \mathrm{e}-02$ & $2.786808620707405 \mathrm{e}-03$ \\
\hline$Z=29$ & $\sigma_{e}=1.9144050 \mathrm{e}-03$ & & $\sigma_{x}=1.4543323 \mathrm{e}-01$ & \\
\hline $1.752071452121456 \mathrm{e}+00$ & $-4.304105234921245 e+01$ & $4.407059155435739 \mathrm{e}+01$ & $1.868761540881447 \mathrm{e}-02$ & $2.017273247916241 \mathrm{e}-04$ \\
\hline $6.187504979861871 \mathrm{e}+00$ & $1.002662636289766 \mathrm{e}+00$ & $9.853843113530303 \mathrm{e}-01$ & $3.029847039161176 \mathrm{e}-02$ & $2.558555987488791 \mathrm{e}-03$ \\
\hline $\mathrm{Z}=30$ & $\sigma_{e}=1.7646653 \mathrm{e}-03$ & & $\sigma_{x}=1.4177450 \mathrm{e}-01$ & \\
\hline $2.466371104994591 \mathrm{e}+00$ & $-6.146785413325375 e+01$ & $6.201769452374813 e+01$ & $1.641601739314162 \mathrm{e}-02$ & $1.724871175953809 \mathrm{e}-04$ \\
\hline $4.910280784938159 \mathrm{e}+00$ & $9.678985203229922 \mathrm{e}-01$ & $9.512838347753555 \mathrm{e}-01$ & $2.696009676675663 \mathrm{e}-02$ & $2.341096110462537 \mathrm{e}-03$ \\
\hline $\mathrm{Z}=31$ & $\sigma_{e}=2.5723604 \mathrm{e}-03$ & & $\sigma_{x}=1.4628622 \mathrm{e}-01$ & \\
\hline $2.760102031084283 \mathrm{e}+00$ & $-3.444526142074679 \mathrm{e}+01$ & $3.522622672440163 \mathrm{e}+01$ & $1.320671969994199 \mathrm{e}-02$ & $1.259455609282457 \mathrm{e}-04$ \\
\hline $6.101282245376627 \mathrm{e}+00$ & $7.651433135534649 \mathrm{e}-01$ & 7.513286233828597e-01 & $2.248796343172512 \mathrm{e}-02$ & $2.067373742787123 \mathrm{e}-03$ \\
\hline $\mathrm{Z}=32$ & $\sigma_{e}=2.4353339 \mathrm{e}-03$ & & $\sigma_{x}=1.4415421 \mathrm{e}-01$ & \\
\hline $3.182416352600000 \mathrm{e}+00$ & $-5.245140378111667 \mathrm{e}+01$ & $5.296908271622185 \mathrm{e}+01$ & $1.140961685918648 \mathrm{e}-02$ & $9.509543581450579 \mathrm{e}-05$ \\
\hline $5.017190408609147 \mathrm{e}+00$ & $7.123957644377982 \mathrm{e}-01$ & $7.022801925281940 \mathrm{e}-01$ & $1.967472956940154 \mathrm{e}-02$ & $1.841466144940115 \mathrm{e}-03$ \\
\hline $\mathrm{Z}=33$ & $\sigma_{e}=2.3192024 \mathrm{e}-03$ & & $\sigma_{x}=1.4238220 \mathrm{e}-01$ & \\
\hline $3.456429691196040 \mathrm{e}+00$ & $-3.331760444317212 \mathrm{e}+01$ & $3.357121938553322 \mathrm{e}+01$ & $9.790022956403421 \mathrm{e}-03$ & $6.534348622651725 \mathrm{e}-05$ \\
\hline $4.013580160329452 \mathrm{e}+00$ & $6.623557780506291 \mathrm{e}-01$ & $6.457719410560738 \mathrm{e}-01$ & $1.709193532310115 \mathrm{e}-02$ & $1.603016028394201 \mathrm{e}-03$ \\
\hline$Z=34$ & $\sigma_{e}=2.3096465 \mathrm{e}-03$ & & $\sigma_{x}=1.4026158 \mathrm{e}-01$ & \\
\hline $3.649050478019264 \mathrm{e}+00$ & $-4.368516622212386 e+01$ & $4.369202886011548 \mathrm{e}+01$ & $8.449022841991444 \mathrm{e}-03$ & $3.786114741100382 \mathrm{e}-05$ \\
\hline $3.250432671125934 \mathrm{e}+00$ & $6.096662016502890 \mathrm{e}-01$ & $5.969713008021232 \mathrm{e}-01$ & $1.485545127403623 \mathrm{e}-02$ & $1.336255873565630 \mathrm{e}-03$ \\
\hline $\mathrm{Z}=35$ & $\sigma_{e}=2.2468181 \mathrm{e}-03$ & & $\sigma_{x}=1.3762379 \mathrm{e}-01$ & \\
\hline $3.838463122242895 \mathrm{e}+00$ & $-5.227234710112339 e+01$ & $5.198612794956596 \mathrm{e}+01$ & $7.339559893380448 \mathrm{e}-03$ & $1.646942079513399 \mathrm{e}-05$ \\
\hline $2.611894705732323 \mathrm{e}+00$ & $5.661950628747182 \mathrm{e}-01$ & $5.552793266998739 \mathrm{e}-01$ & $1.297464694324407 \mathrm{e}-02$ & $1.029865368558757 \mathrm{e}-03$ \\
\hline$Z=36$ & $\sigma_{e}=2.1386880 \mathrm{e}-03$ & & $\sigma_{x}=1.3573200 \mathrm{e}-01$ & \\
\hline $4.025410303108274 \mathrm{e}+00$ & $-4.630423320789298 \mathrm{e}+01$ & $4.572136819041012 \mathrm{e}+01$ & $6.353379596253157 \mathrm{e}-03$ & $1.334778452557430 \mathrm{e}-06$ \\
\hline $2.136483814437400 \mathrm{e}+00$ & $5.265911664541312 \mathrm{e}-01$ & $5.141367844645776 \mathrm{e}-01$ & $1.128072422420068 \mathrm{e}-02$ & $4.880898579409704 \mathrm{e}-04$ \\
\hline $\mathrm{Z}=37$ & $\sigma_{e}=8.0194968 \mathrm{e}-03$ & & $\sigma_{x}=1.8553060 \mathrm{e}-01$ & \\
\hline $3.389753515953940 \mathrm{e}+00$ & $2.143483486791724 \mathrm{e}+00$ & $3.543226035109782 \mathrm{e}-01$ & $3.740093400856642 \mathrm{e}-03$ & $3.003425023422297 \mathrm{e}-07$ \\
\hline $2.057448143681711 \mathrm{e}+01$ & $1.910799452185164 \mathrm{e}+00$ & $1.974105893966039 \mathrm{e}-01$ & $8.134594653439889 \mathrm{e}-03$ & $2.926857910569511 \mathrm{e}-04$ \\
\hline$Z=38$ & $\sigma_{e}=8.1077496 \mathrm{e}-03$ & & $\sigma_{x}=1.7693604 \mathrm{e}-01$ & \\
\hline $4.770925092998260 \mathrm{e}+00$ & $1.475978501552834 \mathrm{e}+00$ & $3.044513555441887 \mathrm{e}-01$ & $3.594749819167283 \mathrm{e}-03$ & $3.000855492282790 \mathrm{e}-07$ \\
\hline $1.336688813304128 \mathrm{e}+01$ & $1.337383795771967 \mathrm{e}+00$ & $1.775323694377828 \mathrm{e}-01$ & $7.791050330018268 \mathrm{e}-03$ & $2.822551396485372 \mathrm{e}-04$ \\
\hline$Z=39$ & $\sigma_{e}=6.3536332 \mathrm{e}-03$ & & $\sigma_{x}=1.7286109 \mathrm{e}-01$ & \\
\hline $4.607210198752340 \mathrm{e}+00$ & $1.428018510398403 \mathrm{e}+00$ & $2.955810455777538 \mathrm{e}-01$ & $3.389978408528951 \mathrm{e}-03$ & $2.668629749690224 \mathrm{e}-07$ \\
\hline $1.086869055571519 \mathrm{e}+01$ & $1.311374558731241 \mathrm{e}+00$ & $1.680228707847112 \mathrm{e}-01$ & $7.359645453504783 \mathrm{e}-03$ & $2.623432810213873 \mathrm{e}-04$ \\
\hline$Z=40$ & $\sigma_{e}=5.2384796 \mathrm{e}-03$ & & $\sigma_{x}=1.7438775 \mathrm{e}-01$ & \\
\hline $4.311754534068716 \mathrm{e}+00$ & $1.493315780393395 \mathrm{e}+00$ & $2.812360501288841 \mathrm{e}-01$ & $3.093377384557471 \mathrm{e}-03$ & $2.580244485125306 \mathrm{e}-07$ \\
\hline $9.458965805174902 \mathrm{e}+00$ & $1.330636228452456 \mathrm{e}+00$ & $1.565068714444536 \mathrm{e}-01$ & $6.824105238117352 \mathrm{e}-03$ & $2.498188791107023 \mathrm{e}-04$ \\
\hline$Z=41$ & $\sigma_{e}=3.9366986 \mathrm{e}-03$ & & $\sigma_{x}=1.8366741 \mathrm{e}-01$ & \\
\hline $3.111790391134954 \mathrm{e}+00$ & $2.202590609458031 \mathrm{e}+00$ & $2.703307749100209 \mathrm{e}-01$ & $2.687991947510981 \mathrm{e}-03$ & $2.325494833477792 \mathrm{e}-07$ \\
\hline $1.069031413430237 \mathrm{e}+01$ & $1.653163561589331 \mathrm{e}+00$ & $1.451151857189699 \mathrm{e}-01$ & $6.139563515828505 \mathrm{e}-03$ & $2.322402359376725 \mathrm{e}-04$ \\
\hline$Z=42$ & $\sigma_{e}=3.7283802 \mathrm{e}-03$ & & $\sigma_{x}=1.9131949 \mathrm{e}-01$ & \\
\hline $2.831059684320536 \mathrm{e}+00$ & $2.348581374896745 \mathrm{e}+00$ & $2.451058884296964 \mathrm{e}-01$ & $2.352821082185159 \mathrm{e}-03$ & $2.312708383438592 \mathrm{e}-07$ \\
\hline $1.043571957589507 \mathrm{e}+01$ & $1.604828686745972 \mathrm{e}+00$ & $1.316969347746069 \mathrm{e}-01$ & $5.549779013833459 \mathrm{e}-03$ & $2.227474637648977 \mathrm{e}-04$ \\
\hline$Z=43$ & $\sigma_{e}=3.6547115 \mathrm{e}-03$ & & $\sigma_{x}=1.9921427 \mathrm{e}-01$ & \\
\hline $2.571798593233526 \mathrm{e}+00$ & $2.456337419572030 \mathrm{e}+00$ & $2.206584408813715 \mathrm{e}-01$ & $2.055314180124382 \mathrm{e}-03$ & $2.321329477936922 \mathrm{e}-07$ \\
\hline $1.016431171313775 \mathrm{e}+01$ & $1.534419192445505 \mathrm{e}+00$ & $1.191386197541104 \mathrm{e}-01$ & $5.018532408829888 \mathrm{e}-03$ & $2.145903791208612 \mathrm{e}-04$ \\
\hline$Z=44$ & $\sigma_{e}=3.6531634 \mathrm{e}-03$ & & $\sigma_{x}=2.0792644 \mathrm{e}-01$ & \\
\hline $2.332300303539466 \mathrm{e}+00$ & $2.535780254890496 \mathrm{e}+00$ & $1.982080421360260 \mathrm{e}-01$ & $1.761201304600535 \mathrm{e}-03$ & $1.981294115460245 \mathrm{e}-07$ \\
\hline $9.921674601599595 \mathrm{e}+00$ & $1.455856688087881 \mathrm{e}+00$ & $1.076821878733493 \mathrm{e}-01$ & $4.472435456549673 e-03$ & $1.965747162324994 \mathrm{e}-04$ \\
\hline$Z=45$ & $\sigma_{e}=3.7004587 \mathrm{e}-03$ & & $\sigma_{x}=2.1682907 \mathrm{e}-01$ & \\
\hline $2.113525348594700 \mathrm{e}+00$ & $2.586363161550138 \mathrm{e}+00$ & $1.770639138636865 \mathrm{e}-01$ & $1.497370510030569 \mathrm{e}-03$ & $2.054814455556224 \mathrm{e}-07$ \\
\hline $9.659137258597623 \mathrm{e}+00$ & $1.371066569343296 \mathrm{e}+00$ & $9.703530275846488 \mathrm{e}-02$ & $3.971285090887921 \mathrm{e}-03$ & $1.913551907377983 \mathrm{e}-04$ \\
\hline$Z=46$ & $\sigma_{e}=3.5196889 \mathrm{e}-03$ & & $\sigma_{x}=2.1842007 \mathrm{e}-01$ & \\
\hline $6.421597961826873 \mathrm{e}-01$ & $2.979148144263289 \mathrm{e}+00$ & $1.681544260042708 \mathrm{e}-01$ & $1.337442138784078 \mathrm{e}-03$ & $1.914109682468616 \mathrm{e}-07$ \\
\hline $5.974797502634061 \mathrm{e}+00$ & $1.433594325412777 \mathrm{e}+00$ & $9.098684011726770 \mathrm{e}-02$ & $3.624101371161622 \mathrm{e}-03$ & $1.806889144160450 \mathrm{e}-04$ \\
\hline $\mathrm{Z}=47$ & $\sigma_{e}=3.8975730 \mathrm{e}-03$ & & $\sigma_{x}=2.3518818 \mathrm{e}-01$ & \\
\hline $1.553172166803896 \mathrm{e}+00$ & $2.639303646999888 \mathrm{e}+00$ & $1.420154869567882 \mathrm{e}-01$ & $1.008504600997609 \mathrm{e}-03$ & $1.946384299730244 \mathrm{e}-07$ \\
\hline $8.156202357589566 \mathrm{e}+00$ & $1.216008874818015 \mathrm{e}+00$ & $7.900988649158734 \mathrm{e}-02$ & $2.965479013639490 \mathrm{e}-03$ & $1.745930959191462 \mathrm{e}-04$ \\
\hline $\mathrm{Z}=48$ & $\sigma_{e}=2.5711911 \mathrm{e}-03$ & & $\sigma_{x}=3.0158908 \mathrm{e}-01$ & \\
\hline $6.153078519928602 \mathrm{e}+01$ & $-7.860167412015821 \mathrm{e}+01$ & $2.155012926027705 \mathrm{e}+01$ & $1.376850156641916 \mathrm{e}-01$ & $3.246449309465210 \mathrm{e}-04$ \\
\hline $3.114681025332473 \mathrm{e}+00$ & $2.760169833885745 \mathrm{e}+00$ & $1.935513123247224 \mathrm{e}+00$ & $7.224683472602932 \mathrm{e}-02$ & $1.170016296246845 \mathrm{e}-03$ \\
\hline
\end{tabular}


Table 1 (continued)

\begin{tabular}{|c|c|c|c|c|}
\hline$Z=49$ & $\sigma_{e}=2.9845505 \mathrm{e}-03$ & & $\sigma_{x}=2.8303659 \mathrm{e}-01$ & \\
\hline $4.222321779015246 \mathrm{e}+00$ & $-2.641213183532026 \mathrm{e}+01$ & $2.728528527087469 \mathrm{e}+01$ & $1.216179018841380 \mathrm{e}-01$ & $3.068835463715258 \mathrm{e}-04$ \\
\hline $6.072655104032275 \mathrm{e}+00$ & $1.645501789593879 \mathrm{e}+00$ & $1.522570749395068 \mathrm{e}+00$ & $6.565079146952256 \mathrm{e}-02$ & $1.120938514730356 \mathrm{e}-03$ \\
\hline$Z=50$ & $\sigma_{e}=3.0069115 \mathrm{e}-03$ & & $\sigma_{x}=2.6790907 \mathrm{e}-01$ & \\
\hline $5.142220746420537 \mathrm{e}+00$ & $-2.549454137625767 e+01$ & $2.574144875486019 \mathrm{e}+01$ & $1.117782275921996 \mathrm{e}-01$ & $2.936473847377427 \mathrm{e}-04$ \\
\hline $5.272726364744845 \mathrm{e}+00$ & $1.531949592091483 \mathrm{e}+00$ & $1.402575250400872 \mathrm{e}+00$ & $6.116853872630869 \mathrm{e}-02$ & $1.075608153945223 \mathrm{e}-03$ \\
\hline$Z=51$ & $\sigma_{e}=3.0117661 \mathrm{e}-03$ & & $\sigma_{x}=2.5418207 \mathrm{e}-01$ & \\
\hline $6.241640318318837 \mathrm{e}+00$ & $-9.338687244195522 \mathrm{e}+01$ & $9.263328758298843 \mathrm{e}+01$ & $1.034126922212987 \mathrm{e}-01$ & $2.818484268943907 \mathrm{e}-04$ \\
\hline $4.269841080826875 \mathrm{e}+00$ & $1.394077461402502 \mathrm{e}+00$ & $1.355668549104345 \mathrm{e}+00$ & $5.726679496521998 \mathrm{e}-02$ & $1.033079542820674 \mathrm{e}-03$ \\
\hline$Z=52$ & $\sigma_{e}=2.9812480 \mathrm{e}-03$ & & $\sigma_{x}=2.4168487 \mathrm{e}-01$ & \\
\hline $7.377433018134030 \mathrm{e}+00$ & $-1.260251069316281 \mathrm{e}+02$ & $1.241284050040956 \mathrm{e}+02$ & $9.599783718185698 \mathrm{e}-02$ & $2.710722163988820 \mathrm{e}-04$ \\
\hline $3.469177577838978 \mathrm{e}+00$ & $1.297598108867366 \mathrm{e}+00$ & $1.267710676460660 \mathrm{e}+00$ & $5.377717501794231 \mathrm{e}-02$ & $9.930845770291768 \mathrm{e}-04$ \\
\hline$Z=53$ & $\sigma_{e}=2.9233012 \mathrm{e}-03$ & & $\sigma_{x}=2.3037298 \mathrm{e}-01$ & \\
\hline $9.644006662721232 \mathrm{e}+00$ & $-1.229244353501125 \mathrm{e}+02$ & $1.186825648165673 \mathrm{e}+02$ & $8.950259470255390 \mathrm{e}-02$ & $2.612761214904750 \mathrm{e}-04$ \\
\hline $2.726455453665441 \mathrm{e}+00$ & $1.237234258508660 \mathrm{e}+00$ & $1.200620368722498 \mathrm{e}+00$ & $5.066786822851999 \mathrm{e}-02$ & $9.554378833834973 \mathrm{e}-04$ \\
\hline$Z=54$ & $\sigma_{e}=2.8546677 \mathrm{e}-03$ & & $\sigma_{x}=2.2052685 \mathrm{e}-01$ & \\
\hline $1.554517496748606 \mathrm{e}+01$ & $-1.182410278567446 \mathrm{e}+02$ & $1.080095249631970 \mathrm{e}+02$ & $8.362593419922217 \mathrm{e}-02$ & $2.519918624290739 \mathrm{e}-04$ \\
\hline $2.106373408654927 \mathrm{e}+00$ & $1.208603761295122 \mathrm{e}+00$ & $1.153952705672140 \mathrm{e}+00$ & $4.781893912748243 e-02$ & $9.198862575926131 \mathrm{e}-04$ \\
\hline $\mathrm{Z}=55$ & $\sigma_{e}=7.0955472 \mathrm{e}-03$ & & $\sigma_{x}=2.2435527 \mathrm{e}-01$ & \\
\hline $4.287087391816923 e+00$ & $3.232506654229650 \mathrm{e}+00$ & $6.740295335617063 e-01$ & $6.189080833876976 \mathrm{e}-02$ & $2.356120529521900 \mathrm{e}-04$ \\
\hline $2.265878707985415 \mathrm{e}+01$ & $2.237973864700642 \mathrm{e}+00$ & $3.689955686643163 e-01$ & $4.022665752984844 \mathrm{e}-02$ & $8.837618908780351 \mathrm{e}-04$ \\
\hline$Z=56$ & $\sigma_{e}=7.2969294 \mathrm{e}-03$ & & $\sigma_{x}=2.0878660 \mathrm{e}-01$ & \\
\hline $6.244751873904615 \mathrm{e}+00$ & $2.351722714163885 e+00$ & $4.742793732222520 \mathrm{e}-01$ & $6.381138742868499 \mathrm{e}-02$ & $2.346512805627924 \mathrm{e}-04$ \\
\hline $1.514313541909856 \mathrm{e}+01$ & $1.453790006048140 \mathrm{e}+00$ & $3.208356463878018 \mathrm{e}-01$ & $4.043545320946998 \mathrm{e}-02$ & $8.540811312800327 \mathrm{e}-04$ \\
\hline$Z=57$ & $\sigma_{e}=5.3979477 \mathrm{e}-03$ & & $\sigma_{x}=2.0238122 \mathrm{e}-01$ & \\
\hline $6.097881795995097 \mathrm{e}+00$ & $2.194951647366750 \mathrm{e}+00$ & $5.481727919600226 \mathrm{e}-01$ & $6.166695732226626 \mathrm{e}-02$ & $2.268073558647142 \mathrm{e}-04$ \\
\hline $1.242885443158548 \mathrm{e}+01$ & $1.505359923520235 \mathrm{e}+00$ & $3.337380397171243 \mathrm{e}-01$ & $3.877445534999988 \mathrm{e}-02$ & $8.240498840648670 \mathrm{e}-04$ \\
\hline$Z=58$ & $\sigma_{e}=6.8379926 \mathrm{e}-03$ & & $\sigma_{x}=2.0263934 \mathrm{e}-01$ & \\
\hline $5.795268796472405 \mathrm{e}+00$ & $2.370226641078433 e+00$ & $4.713987569011149 \mathrm{e}-01$ & $5.743682605878668 \mathrm{e}-02$ & $2.189794892596593 \mathrm{e}-04$ \\
\hline $1.428010550582560 \mathrm{e}+01$ & $1.359690157191345 \mathrm{e}+00$ & $3.020173496635141 \mathrm{e}-01$ & $3.664367981470077 \mathrm{e}-02$ & $7.954345265183711 \mathrm{e}-04$ \\
\hline$Z=59$ & $\sigma_{e}=6.6866276 \mathrm{e}-03$ & & $\sigma_{x}=2.0193250 \mathrm{e}-01$ & \\
\hline $5.604062553775258 \mathrm{e}+00$ & $2.357962595618129 \mathrm{e}+00$ & $4.760010985728824 \mathrm{e}-01$ & $5.441233743152471 \mathrm{e}-02$ & $2.114146022051498 \mathrm{e}-04$ \\
\hline $1.395174902305747 \mathrm{e}+01$ & $1.312397549174392 \mathrm{e}+00$ & $2.949337011929565 \mathrm{e}-01$ & $3.486015424623241 \mathrm{e}-02$ & $7.682616826618097 \mathrm{e}-04$ \\
\hline$Z=60$ & $\sigma_{e}=6.5145010 \mathrm{e}-03$ & & $\sigma_{x}=2.0277343 \mathrm{e}-01$ & \\
\hline $5.429083919697703 \mathrm{e}+00$ & $2.336873253608803 \mathrm{e}+00$ & $4.833735541048819 \mathrm{e}-01$ & $5.146549645574790 \mathrm{e}-02$ & $2.037761328637611 \mathrm{e}-04$ \\
\hline $1.365036494276600 \mathrm{e}+01$ & $1.267598413903199 \mathrm{e}+00$ & $2.886106815769299 \mathrm{e}-01$ & $3.312918074466093 e-02$ & $7.423484838012663 \mathrm{e}-04$ \\
\hline$Z=61$ & $\sigma_{e}=6.3721341 \mathrm{e}-03$ & & $\sigma_{x}=2.0476859 \mathrm{e}-01$ & \\
\hline $5.267744450894446 \mathrm{e}+00$ & $2.308558133263050 \mathrm{e}+00$ & $4.932654790260128 \mathrm{e}-01$ & $4.863562797416966 \mathrm{e}-02$ & $1.963088423206752 \mathrm{e}-04$ \\
\hline $1.336020968273946 \mathrm{e}+01$ & $1.225858565869417 \mathrm{e}+00$ & $2.829196321278669 \mathrm{e}-01$ & $3.147353971271379 \mathrm{e}-02$ & $7.176580250696152 \mathrm{e}-04$ \\
\hline$Z=62$ & $\sigma_{e}=6.2860453 \mathrm{e}-03$ & & $\sigma_{x}=2.0850311 \mathrm{e}-01$ & \\
\hline $5.126804285170776 \mathrm{e}+00$ & $2.269255340083806 \mathrm{e}+00$ & $5.042093004533025 \mathrm{e}-01$ & $4.569239924162220 \mathrm{e}-02$ & $1.886750504938862 \mathrm{e}-04$ \\
\hline $1.315815010261290 \mathrm{e}+01$ & $1.181295082433371 \mathrm{e}+00$ & $2.771070382190629 \mathrm{e}-01$ & $2.979576045495446 \mathrm{e}-02$ & $6.940110991990983 \mathrm{e}-04$ \\
\hline
\end{tabular}

$1.315815010261290 \mathrm{e}+01$

$\mathrm{Z}=63$

$4.979623597498092 \mathrm{e}+00$

$1.283926630780338 \mathrm{e}+01$

$\mathrm{Z}=64$

$5.078358300456114 \mathrm{e}+00$

$1.051327254690473 \mathrm{e}+01$

$\mathrm{Z}=65$

$4.711616366573001 \mathrm{e}+00$

$1.231094159485391 \mathrm{e}+01$

$\mathrm{Z}=66$

$4.590755044851466 \mathrm{e}+00$

$1.206567406233699 \mathrm{e}+01$

$\mathrm{Z}=67$

$4.484001106267108 \mathrm{e}+00$

$1.187492506915706 \mathrm{e}+01$

$\mathrm{Z}=68$

$4.376651407869632 \mathrm{e}+00$

$1.166530807171395 \mathrm{e}+01$

$\mathrm{Z}=69$

$4.283083182474195 \mathrm{e}+00$

$1.149619059566834 \mathrm{e}+01$

$\mathrm{Z}=70$

$4.195638407371233 \mathrm{e}+00$

$1.141077504566523 \mathrm{e}+01$

$\mathrm{Z}=71$

$4.356925932963843 \mathrm{e}+00$

$9.294345147185689 \mathrm{e}+00$

$\mathrm{Z}=72$

$4.331384056649235 \mathrm{e}+00$

$7.876844338102884 \mathrm{e}+00$

$\sigma_{e}=6.1315809 \mathrm{e}-03$

$2.241830874556695 \mathrm{e}+00$

$1.147054464204860 \mathrm{e}+00$

$\sigma_{e}=4.8066570 \mathrm{e}-03$

$1.957440271816590 \mathrm{e}+00$

$1.117649412815246 \mathrm{e}+00$

$\sigma_{e}=5.8094970 \mathrm{e}-03$

$2.172619507865789 \mathrm{e}+00$

$1.087437967674925 \mathrm{e}+00$

$\sigma_{e}=5.6545414 \mathrm{e}-03$

$2.135723730365674 \mathrm{e}+00$

$1.058117371158644 \mathrm{e}+00$

$\sigma_{e}=5.5869657 \mathrm{e}-03$

$2.089043470785398 \mathrm{e}+00$

$1.028284937087896 \mathrm{e}+00$

$\sigma_{e}=5.4535962 \mathrm{e}-03$

$2.046451503836341 \mathrm{e}+00$

$1.003147696754953 \mathrm{e}+00$

$\sigma_{e}=5.3444121 \mathrm{e}-03$

$1.995380014537307 \mathrm{e}+00$

$9.770391020188206 \mathrm{e}-01$

$\sigma_{e}=5.3608922 \mathrm{e}-03$

$1.943332859786456 \mathrm{e}+00$

$9.490119244548774 \mathrm{e}-01$

$\sigma_{e}=4.3391045 \mathrm{e}-03$

$1.695892047773159 \mathrm{e}+00$

$9.105000459574200 \mathrm{e}-01$

$\sigma_{e}=3.4342503 \mathrm{e}-03$

$1.527648647286807 \mathrm{e}+00$

$9.425156426277488 \mathrm{e}-01$
$5.129339614028937 \mathrm{e}-01$ $2.703871609352437 \mathrm{e}-01$

5.928259832213751e-01 $2.843867418336752 \mathrm{e}-01$

$5.397176013428657 \mathrm{e}-01$ $2.598049655092755 \mathrm{e}-01$

$5.513555600941525 \mathrm{e}-01$ $2.539944389186618 \mathrm{e}-01$

5.659326183161046e-01 $2.489078079080760 \mathrm{e}-01$

$5.806628605915877 \mathrm{e}-01$

$2.441532926863124 \mathrm{e}-01$

$5.970535713325043 \mathrm{e}-01$ 2.394291329758291e-01

6.124466172854658e-01 $2.349503265353047 \mathrm{e}-01$

6.639045200684705e-01 $2.387465959113764 \mathrm{e}-01$

$7.357959228912380 \mathrm{e}-01$ $2.416994800398060 \mathrm{e}-01$ $\sigma_{x}=2.1227119 \mathrm{e}$

$4.298018484983344 \mathrm{e}-02$ $2.824187149526020 \mathrm{e}-02$ $\sigma_{x}=2.1150578 \mathrm{e}-01$ 4.195020341439256e-02 2.726633276413471e-02 $\sigma_{x}=2.2130310 \mathrm{e}-01$

$3.797960045478111 \mathrm{e}-02$ $2.532899238951897 \mathrm{e}-02$ $\sigma_{x}=2.2619667 \mathrm{e}-01$

$3.560584067185138 \mathrm{e}-02$ $2.395087396496492 \mathrm{e}-02$ $\sigma_{x}=2.3173178 \mathrm{e}-01$ 3.327035902110630e-02 2.258440655321478e-02 $\sigma_{x}=2.3644973 \mathrm{e}-01$

$3.123885283277667 \mathrm{e}-02$ $2.136185800007310 \mathrm{e}-02$ $\sigma_{x}=2.4174434 \mathrm{e}-01$ $2.909516688695599 \mathrm{e}-02$ $2.009122333272558 \mathrm{e}-02$ $\sigma_{x}=2.4690701 \mathrm{e}-01$ $2.715152076949324 \mathrm{e}-02$ $1.890515762809755 \mathrm{e}-02$ $\sigma_{x}=2.4406703 \mathrm{e}-01$ $2.630200187602814 \mathrm{e}-02$ $1.820985425464623 \mathrm{e}-02$ $\sigma_{x}=2.4431816 \mathrm{e}-01$

$2.495263232014026 \mathrm{e}-02$ $1.728998944485090 \mathrm{e}-02$
$1.813816924857876 \mathrm{e}-04$ $6.714866031739924 \mathrm{e}-04$

$1.752410915283168 \mathrm{e}-04$ $6.503108607073037 \mathrm{e}-04$

$1.669237635648417 \mathrm{e}-04$ $6.292785669619112 \mathrm{e}-04$

$1.598240168567898 \mathrm{e}-04$ 6.094827484332881e-04

$1.524456102828921 \mathrm{e}-04$ $5.903744451594245 \mathrm{e}-04$

$1.453748696625357 \mathrm{e}-04$ 5.721103384547666e-04

$1.380647690375216 \mathrm{e}-04$ $5.543771384922663 \mathrm{e}-04$

$1.305947873519297 \mathrm{e}-04$ 5.372336492097111e-04

1.254973184998238e-04 $5.215593719635622 \mathrm{e}-04$

$1.187408525810578 \mathrm{e}-04$ $5.058346313135256 \mathrm{e}-04$ 
Table 1 (continued)

\begin{tabular}{|c|c|c|c|c|}
\hline$Z=73$ & $\sigma_{e}=2.8440987 \mathrm{e}-03$ & & $\sigma_{x}=2.4600873 \mathrm{e}-01$ & \\
\hline $4.197260013196420 \mathrm{e}+00$ & $1.468548067747088 \mathrm{e}+00$ & $7.839312482110882 \mathrm{e}-01$ & $2.324940948643951 \mathrm{e}-02$ & $1.112613586072860 \mathrm{e}-04$ \\
\hline $6.936740248944572 \mathrm{e}+00$ & $1.017252766183483 \mathrm{e}+00$ & $2.389489397150706 \mathrm{e}-01$ & $1.623324330753810 \mathrm{e}-02$ & $4.902390040211490 \mathrm{e}-04$ \\
\hline$Z=74$ & $\sigma_{e}=2.4774664 \mathrm{e}-03$ & & $\sigma_{x}=2.4855995 \mathrm{e}-01$ & \\
\hline $3.976297158190774 \mathrm{e}+00$ & $1.522926842573418 \mathrm{e}+00$ & $7.977062463905613 \mathrm{e}-01$ & $2.136667415586852 \mathrm{e}-02$ & $1.030786893857173 \mathrm{e}-04$ \\
\hline $6.296857792287749 \mathrm{e}+00$ & $1.112899511576911 \mathrm{e}+00$ & $2.310570406784734 \mathrm{e}-01$ & $1.510135455672045 \mathrm{e}-02$ & $4.746824771050258 \mathrm{e}-04$ \\
\hline $\mathrm{Z}=75$ & $\sigma_{e}=2.2869519 \mathrm{e}-03$ & & $\sigma_{x}=2.5120566 \mathrm{e}-01$ & \\
\hline $3.751443814398119 \mathrm{e}+00$ & $1.627688029776327 \mathrm{e}+00$ & $7.817567554542718 \mathrm{e}-01$ & $1.951708039122916 \mathrm{e}-02$ & $9.431998042934275 \mathrm{e}-05$ \\
\hline $5.797546360829538 \mathrm{e}+00$ & $1.182236310777107 \mathrm{e}+00$ & $2.199135860247702 \mathrm{e}-01$ & $1.398104554814566 \mathrm{e}-02$ & $4.591271258298386 \mathrm{e}-04$ \\
\hline$Z=76$ & $\sigma_{e}=2.1814857 \mathrm{e}-03$ & & $\sigma_{x}=2.5480998 \mathrm{e}-01$ & \\
\hline $3.484015173387116 \mathrm{e}+00$ & $1.793779204169655 \mathrm{e}+00$ & $7.448783566919203 e-01$ & $1.754277851624345 \mathrm{e}-02$ & $8.448723515688600 \mathrm{e}-05$ \\
\hline $5.439988460809537 \mathrm{e}+00$ & $1.227921341401281 \mathrm{e}+00$ & $2.062088569929092 \mathrm{e}-01$ & $1.278994017211460 \mathrm{e}-02$ & $4.430322267874786 \mathrm{e}-04$ \\
\hline $\mathrm{Z}=77$ & $\sigma_{e}=2.1716420 \mathrm{e}-03$ & & $\sigma_{x}=2.6718827 \mathrm{e}-01$ & \\
\hline $1.599565781988442 \mathrm{e}+00$ & $2.975344521941205 \mathrm{e}+00$ & $6.950926783668822 \mathrm{e}-01$ & $1.487796274586880 \mathrm{e}-02$ & $6.905495791015833 \mathrm{e}-05$ \\
\hline $5.792444473855675 \mathrm{e}+00$ & $1.553009829732260 \mathrm{e}+00$ & $1.886263359366482 \mathrm{e}-01$ & $1.117634706624533 \mathrm{e}-02$ & $4.227723616555514 \mathrm{e}-04$ \\
\hline $\mathrm{Z}=78$ & $\sigma_{e}=2.2090151 \mathrm{e}-03$ & & $\sigma_{x}=2.7015269 \mathrm{e}-01$ & \\
\hline $2.040215639757283 \mathrm{e}+00$ & $2.899226346248255 \mathrm{e}+00$ & $6.363440838157977 \mathrm{e}-01$ & $1.320719195954083 \mathrm{e}-02$ & $5.673821925001312 \mathrm{e}-05$ \\
\hline $6.658194296096275 \mathrm{e}+00$ & $1.413379237789324 \mathrm{e}+00$ & $1.740001045021790 \mathrm{e}-01$ & $1.006878045302105 \mathrm{e}-02$ & $4.030771056922231 \mathrm{e}-04$ \\
\hline $\mathrm{Z}=79$ & $\sigma_{e}=2.2837831 \mathrm{e}-03$ & & $\sigma_{x}=2.7320354 \mathrm{e}-01$ & \\
\hline $1.675934670648708 \mathrm{e}+00$ & $3.004866029697293 e+00$ & $5.953400131616355 \mathrm{e}-01$ & $1.171631866230948 \mathrm{e}-02$ & 4.296782976398171e-05 \\
\hline $5.522310932114025 \mathrm{e}+00$ & $1.380072230071963 \mathrm{e}+00$ & $1.622292376559454 \mathrm{e}-01$ & $9.018148904165756 \mathrm{e}-03$ & $3.792776674776671 \mathrm{e}-04$ \\
\hline $\mathrm{Z}=80$ & $\sigma_{e}=2.3404236 \mathrm{e}-03$ & & $\sigma_{x}=2.7007986 \mathrm{e}-01$ & \\
\hline $2.235228504431053 \mathrm{e}+00$ & $2.682766386519949 \mathrm{e}+00$ & $5.551949262124333 \mathrm{e}-01$ & $1.072733543662587 \mathrm{e}-02$ & $3.284739920462629 \mathrm{e}-05$ \\
\hline $5.020309889602403 \mathrm{e}+00$ & $1.230775905837777 \mathrm{e}+00$ & $1.522481229928636 \mathrm{e}-01$ & $8.283991169062104 \mathrm{e}-03$ & $3.562419389317589 \mathrm{e}-04$ \\
\hline $\mathrm{Z}=81$ & $\sigma_{e}=2.0915554 \mathrm{e}-03$ & & $\sigma_{x}=2.6589399 \mathrm{e}-01$ & \\
\hline $2.803427374125100 \mathrm{e}+00$ & $2.718827879660200 \mathrm{e}+00$ & $5.224759153919996 \mathrm{e}-01$ & $9.845378369710420 \mathrm{e}-03$ & $2.345245265083162 \mathrm{e}-05$ \\
\hline $6.558768728045342 \mathrm{e}+00$ & $1.169724225166679 \mathrm{e}+00$ & $1.435566918001781 \mathrm{e}-01$ & $7.619765262300398 \mathrm{e}-03$ & $3.296276739029854 \mathrm{e}-04$ \\
\hline $\mathrm{Z}=82$ & $\sigma_{e}=2.1232856 \mathrm{e}-03$ & & $\sigma_{x}=2.6373903 \mathrm{e}-01$ & \\
\hline $3.608610209977714 \mathrm{e}+00$ & $2.450567747371983 \mathrm{e}+00$ & $4.786395001072570 \mathrm{e}-01$ & $8.872142351692152 \mathrm{e}-03$ & $1.040019329094673 \mathrm{e}-05$ \\
\hline $6.581625946219628 \mathrm{e}+00$ & $1.027728526605885 \mathrm{e}+00$ & $1.335336806347209 \mathrm{e}-01$ & $6.848612389484298 \mathrm{e}-03$ & $2.763888754566660 \mathrm{e}-04$ \\
\hline $\mathrm{Z}=83$ & $\sigma_{e}=2.1765756 \mathrm{e}-03$ & & $\sigma_{x}=2.6034658 \mathrm{e}-01$ & \\
\hline $4.242099010573159 \mathrm{e}+00$ & $2.099943420558279 \mathrm{e}+00$ & $4.328366313799229 \mathrm{e}-01$ & $8.020215703810592 \mathrm{e}-03$ & $7.217850308513403 \mathrm{e}-07$ \\
\hline $5.752801155360800 \mathrm{e}+00$ & $8.739014891954615 \mathrm{e}-01$ & $1.235999561805247 \mathrm{e}-01$ & $6.176003330585702 \mathrm{e}-03$ & $1.414951776172309 \mathrm{e}-04$ \\
\hline$Z=84$ & $\sigma_{e}=2.1603237 \mathrm{e}-03$ & & $\sigma_{x}=2.4991426 \mathrm{e}-01$ & \\
\hline $4.636200956689623 \mathrm{e}+00$ & $1.780633114269871 \mathrm{e}+00$ & $4.037304439527385 \mathrm{e}-01$ & $7.685395546686740 \mathrm{e}-03$ & $8.954159028076436 \mathrm{e}-08$ \\
\hline $4.888252997809829 \mathrm{e}+00$ & $7.563105268800747 \mathrm{e}-01$ & $1.173023644522492 \mathrm{e}-01$ & $5.930343942428942 \mathrm{e}-03$ & 7.666686638730287e-05 \\
\hline$Z=85$ & $\sigma_{e}=2.2646811 \mathrm{e}-03$ & & $\sigma_{x}=2.3907733 \mathrm{e}-01$ & \\
\hline $4.965922505950705 \mathrm{e}+00$ & $1.438155615070337 \mathrm{e}+00$ & $3.712992005792981 \mathrm{e}-01$ & $7.472564284502011 \mathrm{e}-03$ & $1.141152841525851 \mathrm{e}-07$ \\
\hline $4.091293787874963 \mathrm{e}+00$ & $6.292289966025513 \mathrm{e}-01$ & $1.110966786955352 \mathrm{e}-01$ & $5.772350103477618 \mathrm{e}-03$ & $8.085118938793825 \mathrm{e}-05$ \\
\hline$Z=86$ & $\sigma_{e}=2.7438639 \mathrm{e}-03$ & & $\sigma_{x}=2.3054398 \mathrm{e}-01$ & \\
\hline $5.306156144750332 \mathrm{e}+00$ & $1.117331602360721 \mathrm{e}+00$ & $3.158587231108543 \mathrm{e}-01$ & $7.203422423102100 \mathrm{e}-03$ & $1.073553305458635 \mathrm{e}-07$ \\
\hline $3.488007354816557 \mathrm{e}+00$ & 4.811907411497712e-01 & $1.018744679457531 \mathrm{e}-01$ & $5.592453744170787 \mathrm{e}-03$ & 7.795066735407367e-05 \\
\hline$Z=87$ & $\sigma_{e}=4.6960058 \mathrm{e}-03$ & & $\sigma_{x}=2.1038024 \mathrm{e}-01$ & \\
\hline $4.520533990423360 \mathrm{e}+00$ & $4.106953979091246 \mathrm{e}+00$ & $7.139468785037285 e-01$ & $1.692940276874539 \mathrm{e}-02$ & $8.574921291917244 \mathrm{e}-05$ \\
\hline $1.944822342329488 \mathrm{e}+01$ & $1.898246731559969 \mathrm{e}+00$ & $1.695535635953418 \mathrm{e}-01$ & $1.148195675489342 \mathrm{e}-02$ & $3.461220382579827 \mathrm{e}-04$ \\
\hline$Z=88$ & $\sigma_{e}=4.6272870 \mathrm{e}-03$ & & $\sigma_{x}=1.8975618 \mathrm{e}-01$ & \\
\hline $6.524010720018733 \mathrm{e}+00$ & $3.207870807456613 \mathrm{e}+00$ & $5.404787743496377 \mathrm{e}-01$ & $8.782788068988532 \mathrm{e}-03$ & $6.910106029491315 \mathrm{e}-06$ \\
\hline $1.400925542989749 \mathrm{e}+01$ & $1.326350359616653 \mathrm{e}+00$ & $1.314085683860361 \mathrm{e}-01$ & $6.286474345204096 \mathrm{e}-03$ & $2.278399814589510 \mathrm{e}-04$ \\
\hline$Z=89$ & $\sigma_{e}=3.4516587 \mathrm{e}-03$ & & $\sigma_{x}=1.8451020 \mathrm{e}-01$ & \\
\hline $6.896028535596191 \mathrm{e}+00$ & $2.835141545365232 \mathrm{e}+00$ & $5.035068818888151 \mathrm{e}-01$ & $8.022945109667069 \mathrm{e}-03$ & $9.204009547587397 \mathrm{e}-08$ \\
\hline $1.107638256703987 \mathrm{e}+01$ & $1.171326162905030 \mathrm{e}+00$ & $1.234946513917999 \mathrm{e}-01$ & $5.735155957904971 \mathrm{e}-03$ & $7.197075418025232 \mathrm{e}-05$ \\
\hline$Z=90$ & $\sigma_{e}=2.5645470 \mathrm{e}-03$ & & $\sigma_{x}=1.7847619 \mathrm{e}-01$ & \\
\hline $7.093749001626302 \mathrm{e}+00$ & $2.529123739031940 \mathrm{e}+00$ & $4.821078198888890 \mathrm{e}-01$ & $7.719366741451110 \mathrm{e}-03$ & $7.271141994250414 \mathrm{e}-08$ \\
\hline $9.094737951659447 \mathrm{e}+00$ & $1.063916670331612 \mathrm{e}+00$ & $1.186194466218779 \mathrm{e}-01$ & $5.539457088950347 \mathrm{e}-03$ & $6.584947305284627 \mathrm{e}-05$ \\
\hline $\mathrm{Z}=91$ & $\sigma_{e}=3.0610380 \mathrm{e}-03$ & & $\sigma_{x}=1.7782903 \mathrm{e}-01$ & \\
\hline $6.434013247972842 \mathrm{e}+00$ & $2.970999705357888 \mathrm{e}+00$ & $4.607966517878480 \mathrm{e}-01$ & $7.240331257754553 e-03$ & $6.362366643091279 \mathrm{e}-08$ \\
\hline $1.025968513072979 \mathrm{e}+01$ & $1.131774523061633 \mathrm{e}+00$ & $1.127759353623823 e-01$ & $5.274595833531321 \mathrm{e}-03$ & $6.192638240887597 \mathrm{e}-05$ \\
\hline$Z=92$ & $\sigma_{e}=2.9086967 \mathrm{e}-03$ & & $\sigma_{x}=1.7864173 \mathrm{e}-01$ & \\
\hline $6.210708267840199 \mathrm{e}+00$ & $3.039344538256235 \mathrm{e}+00$ & $4.373399844391628 \mathrm{e}-01$ & $6.807147641473166 \mathrm{e}-03$ & $6.182293277428513 \mathrm{e}-08$ \\
\hline $1.002141058591628 \mathrm{e}+01$ & $1.103998801558834 \mathrm{e}+00$ & $1.072189731624539 \mathrm{e}-01$ & $5.024321308506824 \mathrm{e}-03$ & $6.005069941971958 \mathrm{e}-05$ \\
\hline$Z=93$ & $\sigma_{e}=2.7901195 \mathrm{e}-03$ & & $\sigma_{x}=1.8111339 \mathrm{e}-01$ & \\
\hline $6.004315983089865 \mathrm{e}+00$ & $3.094316545314180 \mathrm{e}+00$ & $4.128084877164170 \mathrm{e}-01$ & $6.408916578800669 \mathrm{e}-03$ & $6.730073762160754 \mathrm{e}-08$ \\
\hline $9.817930467191061 \mathrm{e}+00$ & $1.069787050680294 \mathrm{e}+00$ & $1.016352914486458 \mathrm{e}-01$ & $4.795248468887250 \mathrm{e}-03$ & $6.028065987264629 \mathrm{e}-05$ \\
\hline$Z=94$ & $\sigma_{e}=3.1014809 \mathrm{e}-03$ & & $\sigma_{x}=1.8133996 \mathrm{e}-01$ & \\
\hline $5.200617168103042 \mathrm{e}+00$ & $3.498494403671440 \mathrm{e}+00$ & $4.054149311313203 e-01$ & $6.223437008265299 \mathrm{e}-03$ & $6.008593295434409 \mathrm{e}-08$ \\
\hline $1.120383101944067 \mathrm{e}+01$ & $1.128463695469282 \mathrm{e}+00$ & $9.893334726861316 \mathrm{e}-02$ & 4.659174319779507e-03 & $5.725906389623341 \mathrm{e}-05$ \\
\hline$Z=95$ & $\sigma_{e}=2.9442501 \mathrm{e}-03$ & & $\sigma_{x}=1.8630618 \mathrm{e}-01$ & \\
\hline $5.025338600360291 \mathrm{e}+00$ & $3.518439882851541 \mathrm{e}+00$ & $3.819503494462722 \mathrm{e}-01$ & $5.821102080962110 \mathrm{e}-03$ & $6.526093388297119 \mathrm{e}-08$ \\
\hline $1.097721906976287 \mathrm{e}+01$ & $1.084772148326308 \mathrm{e}+00$ & $9.367468748538359 \mathrm{e}-02$ & $4.426823468734856 \mathrm{e}-03$ & $5.738568370505115 \mathrm{e}-05$ \\
\hline$Z=96$ & $\sigma_{e}=2.4472685 \mathrm{e}-03$ & & $\sigma_{x}=1.9515674 \mathrm{e}-01$ & \\
\hline $5.346561002606197 \mathrm{e}+00$ & $3.224684665609108 \mathrm{e}+00$ & $3.494617526254452 \mathrm{e}-01$ & $5.292517567488151 \mathrm{e}-03$ & $6.159176302362374 \mathrm{e}-08$ \\
\hline $9.231183797524494 \mathrm{e}+00$ & $9.728362291938352 \mathrm{e}-01$ & $8.705962209665623 e-02$ & $4.130119644650323 \mathrm{e}-03$ & $5.494638944496757 \mathrm{e}-05$ \\
\hline
\end{tabular}


Table 1 (continued)

\begin{tabular}{|c|c|c|c|c|}
\hline$Z=97$ & $\sigma_{e}=2.3667676 \mathrm{e}-03$ & & $\sigma_{x}=2.0262210 \mathrm{e}-01$ & \\
\hline $5.225823503340047 \mathrm{e}+00$ & $3.228188739952953 \mathrm{e}+00$ & $3.270988788238514 \mathrm{e}-01$ & $4.888825755922390 \mathrm{e}-03$ & $5.212722694193633 \mathrm{e}-08$ \\
\hline $9.071357066187183 \mathrm{e}+00$ & $9.311242774566348 \mathrm{e}-01$ & $8.207206020598455 \mathrm{e}-02$ & $3.890296559934166 \mathrm{e}-03$ & $5.103679766366416 \mathrm{e}-05$ \\
\hline$Z=98$ & $\sigma_{e}=2.6046538 \mathrm{e}-03$ & & $\sigma_{x}=2.0354840 \mathrm{e}-01$ & \\
\hline $4.586412477535479 \mathrm{e}+00$ & $3.518695956651012 \mathrm{e}+00$ & $3.192617142012054 \mathrm{e}-01$ & $4.729796479856258 \mathrm{e}-03$ & $5.513244808062041 \mathrm{e}-08$ \\
\hline $1.031861020923352 \mathrm{e}+01$ & $9.572788378292328 \mathrm{e}-01$ & $7.968074314438807 \mathrm{e}-02$ & $3.771931999968489 \mathrm{e}-03$ & $5.097508603760111 \mathrm{e}-05$ \\
\hline$Z=99$ & $\sigma_{e}=2.4717479 \mathrm{e}-03$ & & $\sigma_{x}=2.0891883 \mathrm{e}-01$ & \\
\hline $4.457994806754126 \mathrm{e}+00$ & $3.508672126376623 \mathrm{e}+00$ & $3.013753805283154 \mathrm{e}-01$ & $4.407637462144819 \mathrm{e}-03$ & $4.887879032285916 \mathrm{e}-08$ \\
\hline $1.008906933834013 \mathrm{e}+01$ & $9.194464473616226 \mathrm{e}-01$ & $7.564191777863293 e-02$ & $3.570264651248093 \mathrm{e}-03$ & $4.804951743958113 \mathrm{e}-05$ \\
\hline$Z=100$ & $\sigma_{e}=2.4129784 \mathrm{e}-03$ & & $\sigma_{x}=2.1752401 \mathrm{e}-01$ & \\
\hline $4.338975764011709 \mathrm{e}+00$ & $3.491850988964034 \mathrm{e}+00$ & $2.814710990162868 \mathrm{e}-01$ & $4.002092714990461 \mathrm{e}-03$ & $5.529298081955140 \mathrm{e}-08$ \\
\hline $9.963309372238362 \mathrm{e}+00$ & $8.780839569829744 \mathrm{e}-01$ & $7.116371157687776 \mathrm{e}-02$ & $3.321524042150777 \mathrm{e}-03$ & $4.851031794603728 \mathrm{e}-05$ \\
\hline$Z=101$ & $\sigma_{e}=2.3056064 \mathrm{e}-03$ & & $\sigma_{x}=2.2300363 \mathrm{e}-01$ & \\
\hline $4.227294704031012 \mathrm{e}+00$ & $3.472492275106616 \mathrm{e}+00$ & $2.648222294968986 \mathrm{e}-01$ & $3.690728778331929 \mathrm{e}-03$ & $6.258714262002473 \mathrm{e}-08$ \\
\hline $9.724006020400314 \mathrm{e}+00$ & $8.428737759602104 \mathrm{e}-01$ & $6.735347439748511 \mathrm{e}-02$ & $3.123646062633208 \mathrm{e}-03$ & $4.912170970176192 \mathrm{e}-05$ \\
\hline$Z=102$ & $\sigma_{e}=2.3045333 \mathrm{e}-03$ & & $\sigma_{x}=2.3171091 \mathrm{e}-01$ & \\
\hline $4.109517024430204 \mathrm{e}+00$ & $3.457991325227507 \mathrm{e}+00$ & $2.470873512223867 \mathrm{e}-01$ & $3.304239209409952 \mathrm{e}-03$ & $5.991049262319316 \mathrm{e}-08$ \\
\hline $9.677359945101202 \mathrm{e}+00$ & $8.069400424708172 \mathrm{e}-01$ & $6.328154369541671 \mathrm{e}-02$ & $2.875446396412092 \mathrm{e}-03$ & 4.706791536975981e-05 \\
\hline$Z=103$ & $\sigma_{e}=1.6260491 \mathrm{e}-03$ & & $\sigma_{x}=2.4427470 \mathrm{e}-01$ & \\
\hline $4.521474211983788 \mathrm{e}+00$ & $3.202129855878044 \mathrm{e}+00$ & $2.230287269564517 \mathrm{e}-01$ & $2.817164538920297 \mathrm{e}-03$ & $4.064279540386205 \mathrm{e}-08$ \\
\hline $8.283099068611421 \mathrm{e}+00$ & $7.319189581253939 \mathrm{e}-01$ & $5.809427730186550 \mathrm{e}-02$ & $2.561680160474449 \mathrm{e}-03$ & $4.038165155290065 \mathrm{e}-05$ \\
\hline
\end{tabular}

$$
b_{i}^{\prime}=b_{i}+r \times d_{i},
$$

where $r$ is a uniform random number generated in the interval $[-1,1]$ and $d_{i}$ is the neighbourhood range which is varied according to the acceptance rate $p$, as follows:

$$
d_{i}^{\prime}=d_{i} \times g(p)
$$

where

$$
g(p)= \begin{cases}1+c \frac{p-p_{1}}{p_{2}}, & \text { if } p>p_{1} \\ \left(1+c \frac{p_{2}-p}{p_{2}}\right)^{-1}, & \text { if } p<p_{2} \\ 1, & \text { otherwise }\end{cases}
$$

and $p_{1}=0.6, p_{2}=0.4$ and $c=2$.

At a given numerical temperature $T$, the $b^{\prime}$ nonlinear parameters are randomly generated and the $a^{\prime}$ linear coefficients are calculated until they fulfil the constraint conditions. For these sets of coefficients a new cost function $\chi^{\prime 2}$ is calculated. If this new value of cost function is lower than the previous value, then $\mu=\left\{b, a, \chi^{2}\right\}$ are accepted and updated to $\mu^{\prime}$. If the value is higher, then the new point is accepted or rejected using the Metropolis criterion (Metropolis et al., 1953). This process continues $N_{s}$ cycles, and at this stage the acceptance rate is obtained as $p=$ No. accepted points $/ N_{s}$ and the $d_{i}$ values are updated by equation (39). This step is repeated $N_{t}$ cycles, then the temperature is decreased according to the law $T=r_{t} T$, where $r_{t}$ is a cooling parameter in the interval $[0,1]$ and $\mu$ is updated to $\mu^{\mathrm{opt}} . \mu^{\mathrm{opt}}$ is the best configuration that minimizes $\chi^{2}$ after $N_{t} \times N_{s}$ cycles. This process terminates when the temperature is decreased to less than a threshold temperature $T_{\min }$.

The SA can be speeded up as follows. For a given set of coefficients $b_{i}$, the linear least-square procedure guarantees to find one solution to the linear coefficients $a_{i}$. This means that the cost function defined in equation (25) is invariant to all permutations of a given set of $b_{i}$ coefficients. Therefore, we can speed up the search space by setting an inequality constraint for the nonlinear coefficients, let us say $b_{i}>b_{i+1}$, $i=1, \ldots, n_{t}$.

\subsection{Computer program}

A computer program was written in $\mathrm{C}++$ to analytically fit the numerical X-ray and electron scattering factors using $n_{t}$ hydrogen electron scattering factors as basis functions based on the procedure described in the previous section. The uniform pseudo-random numbers that are used in the SA optimization are generated based on the Mersenne Twister algorithm, which for a particular choice of parameters provides a huge repeated period $2^{19937}-1$ (Matsumoto \& Nishimura, 1998). After numerical experiments, we found that satisfactory results for both X-ray and electron scattering factors can be obtained by taking $n_{t}=5$. In the present case, the number of exact constraint conditions is $n_{b}=3$. The accuracy of the SA routine is controlled by six parameters: the initial numerical temperature $T_{0}$, the upper bound of the nonlinear parameters $b_{i}^{\max }$, the cooling parameter $r_{t}$, the number of cycles used for the calculation of the acceptance rate $N_{s}$, the number of cycles after which the neighbourhood range is updated $N_{t}$ and the threshold numerical temperature $T_{\min }$. The SA method is a global optimization technique which statistically guarantees to find an optimal solution when $T_{0}$ is high enough and the system is cooled down sufficiently slowly $r_{t} \rightarrow 1$. Therefore, these parameters are chosen as a result of a compromise between the desired accuracy and the computer time needed to achieve such accuracy. After several numerical experiments reasonable values for the control parameters of the SA are found, which ensures an optimal solution is found that minimized the cost function subject to the constraint conditions:

$$
\begin{array}{cc}
T_{0} & =1000, \\
T_{\min } & =10^{-10}, \\
N_{t} & =200, \\
N_{s} & =30, \\
r_{t} & =0.95, \\
b_{i}^{\max } & =1000 .
\end{array}
$$


Our code reads the following input parameters: the control parameters of the SA [equation (41)], the numerical values of the X-ray and electron scattering factor. In the present work we used the numerical tabulated values of the X-ray and electron scattering factors for all neutral atoms with atomic numbers $Z=2$ through $Z=103$ calculated by Kirkland (1998), which are considered the most accurate. The analytical expressions for the X-ray and electron scattering factor from hydrogen were also fitted to the same parameters as the rest of the atoms in the periodic chart for completeness, although this is not strictly necessary. All these numerical values are tabulated for $g$ values in the range of $0<g<12 \AA^{-1}$ at intervals of $0.05 \AA^{-1} \cdot f_{\mathrm{e}}(g)$ is in units of $\AA$ and $f_{\mathrm{x}}(g)$ is in dimensionless units of electron number.

\section{Results}

The results of the numerical fitting of the X-ray and electron scattering factors with ten parameters for all 103 neutral atoms are given in Table 1 . These parameters are listed for each atomic number $Z$ in the following order:

$$
\begin{array}{ccccc}
Z & \sigma_{\mathrm{e}} & & \sigma_{\mathrm{x}} & \\
a_{1} & a_{2} & a_{3} & a_{4} & a_{5} \\
b_{1} & b_{2} & b_{3} & b_{4} & b_{5}
\end{array}
$$

where $a_{i}$ and $b_{i}$ have units of $\AA$ and $\AA^{2} . \sigma_{\mathrm{e}}$ and $\sigma_{\mathrm{x}}$ are the rootmean-square values of the deviation between the numerical and fitted electron and X-ray scattering factors, respectively.

$$
\begin{aligned}
\sigma_{\mathrm{e}} & =\left\{\frac{1}{n_{g}} \sum_{i=1}^{n_{g}}\left[f_{\mathrm{e} i}-f_{\mathrm{e}}\left(g_{i}\right)\right]^{2}\right\}^{1 / 2} \\
\sigma_{\mathrm{x}} & =\left\{\frac{1}{n_{g}} \sum_{i=1}^{n_{g}}\left[f_{\mathrm{x} i}-f_{\mathrm{x}}\left(g_{i}\right)\right]^{2}\right\}^{1 / 2} .
\end{aligned}
$$

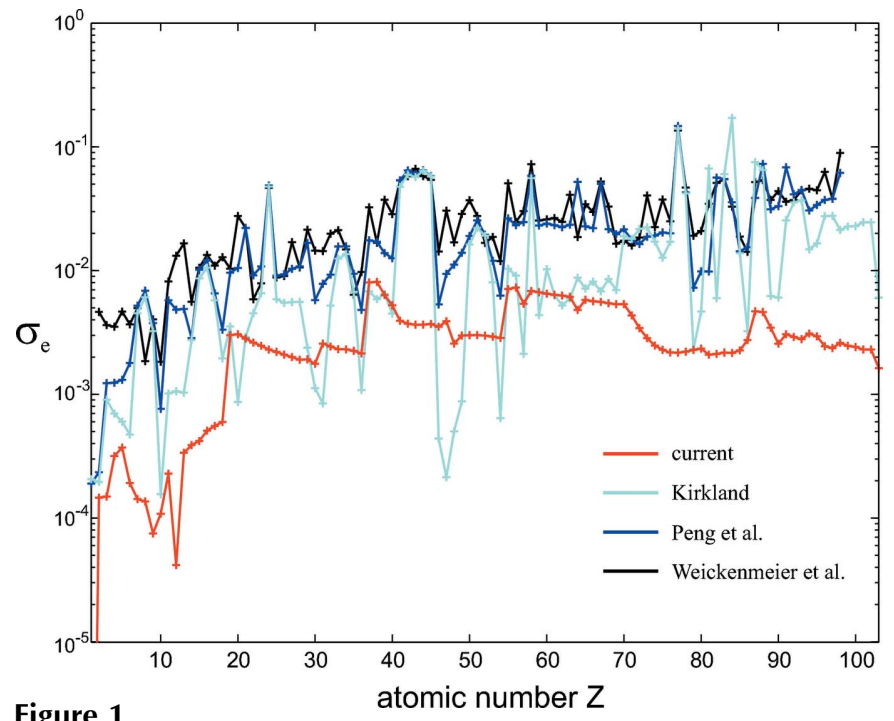

Figure 1

The root-mean-square values of the deviation $\sigma_{\mathrm{e}}$ between the numerical and fitted electron scattering factors versus atomic number $Z$ using four different parameterizations.
The new parameterized electron scattering factor is compared with three different parameterizations, which were fitted using tabulated data of the electron scattering factors up to $12 \AA^{-1}$. For better comparison a different symbol and colour are assigned to each parameterization as follows: Weickenmeier \& Kohl (1991) by WK (black), Peng et al. (1996) by PRDW (blue), Kirkland (1998) by Kl (cyan) and the current one by LD (red). The tabulated values of the X-ray and electron scattering factors numerically calculated by Kirkland (1998) are represented by green markers.

Fig. 1 shows the calculated $\sigma_{\mathrm{e}}$ in logarithmic scale versus the atomic number $Z$. All $f_{\mathrm{e}}(g)$ fittings are compared to the numerical electron scattering data of Kirkland (1998). There is a fitting quality relation among the four $\sigma_{\mathrm{e}}$ which holds for most atomic numbers and it can be expressed as $\sigma_{\mathrm{e}}^{\mathrm{LD}}<\sigma_{\mathrm{e}}^{\mathrm{Kl}}<\sigma_{\mathrm{e}}^{\mathrm{PRDW}}<\sigma_{\mathrm{e}}^{\mathrm{WK}}$. Globally, for all atomic numbers, our proposed parameterization is one order of magnitude better than the last available parameterization (Kirkland, 1998). For the hydrogen atom $\sigma_{\mathrm{e}}=3.2550269 \times 10^{-15}$, which is close to the limit for double-precision floating-point calculations. There are a few points where Kirkland's parameterization gives a lower $\sigma_{\mathrm{e}}$ than our parameterization. The largest difference is found for silver $(Z=47)$. To study the cause of this, we perform a complete comparison among the four different parameterizations. As mentioned in $\$ 1$, the parameterized forms of the electron scattering factor and its

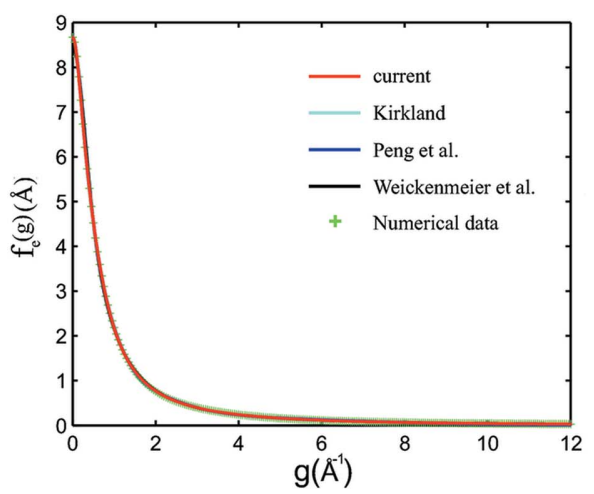

(a)

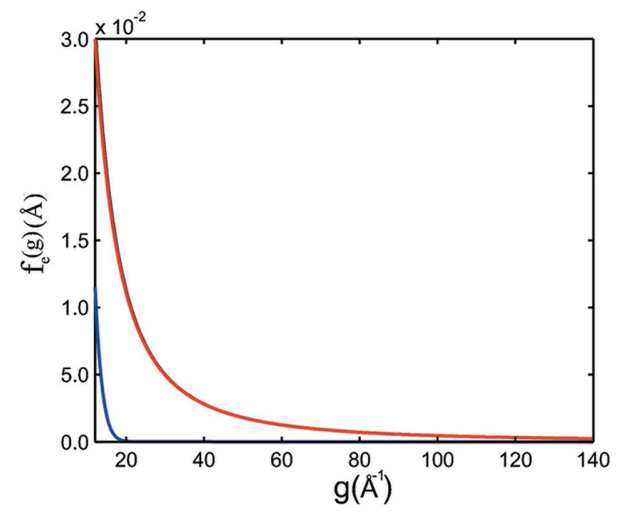

(b)

Figure 2

Comparison of different fittings of the electron scattering factor versus scattering angle for Ag. (a) Interpolated and (b) extrapolated fittings. The green markers are the tabulated numerical values of the electron scattering factor of Kirkland. 
derived quantities have to show the correct behaviour in all ranges. The analytic expressions for the four parameterizations of the electron scattering factor and its derived quantities are calculated in Appendix $A$.

Fig. 2 shows the electron scattering factor versus the threedimensional reciprocal vector $g$ for $\operatorname{Ag}(Z=47)$. Fig. 2(a) shows a comparison of the parameterized and tabulated values of the elastic electron scattering factor. At this scale, all parameterized electron scattering factors are in good agreement with the tabulated electron scattering data. Fig. 2(b) shows the extrapolated values of the parameterized $f_{\mathrm{e}}(g)$ up to $g<140 \AA^{-1}$. The asymptotic behaviour of $f_{\mathrm{e}}(g)$ is obtained by the Mott-Bethe formula, which implies that $f_{\mathrm{e}}(g)$ has to decay proportionally to $1 / g^{2}$ for large $g$ values. $f_{\mathrm{e}}^{\mathrm{PRDW}}(g)$ is the only one that does not show this behaviour for large $g$ values; this is due to the exclusive use of Gaussians as basis functions to express $f_{\mathrm{e}}(g)$ [equation (44)].

Fig. 3 shows the $\mathrm{X}$-ray scattering factor versus the threedimensional reciprocal vector $g$ for $\operatorname{Ag}(Z=47)$. A comparison of the parameterized and tabulated values of the X-ray scattering factor is shown in Fig. 3(a). The calculated $f_{\mathrm{x}}^{\mathrm{Kl}}(g)$ and $f_{\mathrm{X}}^{\mathrm{LD}}(g)$ are in good agreement with the tabulated values of the $\mathrm{X}$-ray scattering factor up to $g<12 \AA^{-1}$. On the other hand, $f_{\mathrm{x}}^{\mathrm{PRDW}}(g)$ shows a large deviation from the tabulated data especially for large frequencies [equation (45)]. $f_{\mathrm{x}}^{\mathrm{WK}}(g)$ shows a small deviation from the tabulated data for low spatial

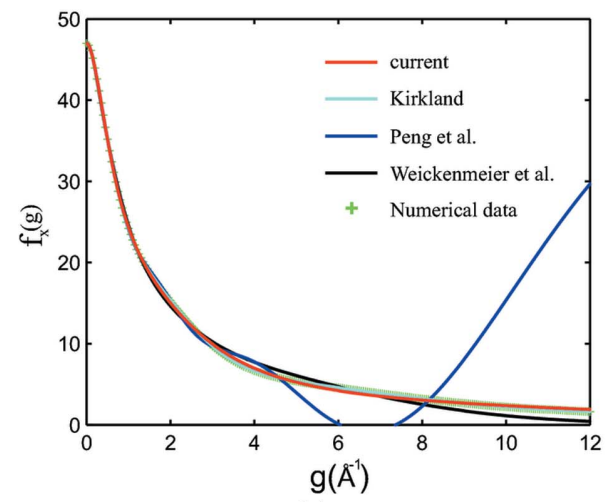

(a)

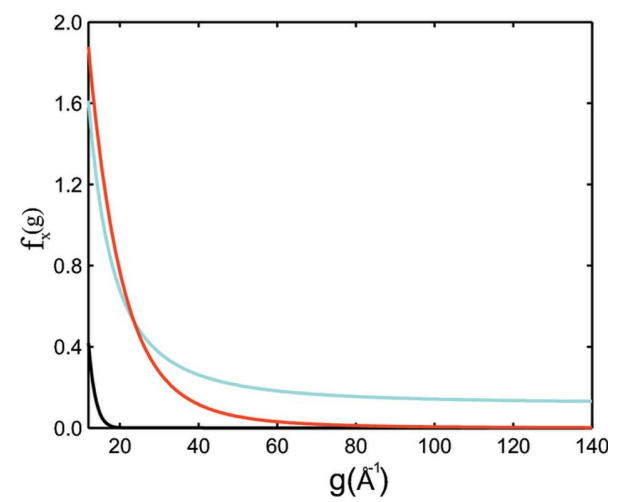

(b)

Figure 3

Comparison of different fittings of the X-ray scattering factor versus scattering angle for Ag. (a) Interpolated and $(b)$ extrapolated fittings. The green markers are the tabulated numerical values of the X-ray scattering factor of Kirkland. frequencies which increases for large spatial frequencies. It is also known that the X-ray scattering factor has to decay to zero for large $g$ values, which is an implication of the Riemann-Lebesgue lemma [equation (18)]. Fig. 3(b) shows the extrapolated values of the parameterized $f_{\mathrm{x}}(g)$ up to $g<140 \AA^{-1} \cdot f_{\mathrm{x}}^{\mathrm{PRDW}}$ is not shown at this scale, because it lies outside of the range of the figure. Both $f_{\mathrm{x}}^{\mathrm{WK}}(g)$ and $f_{\mathrm{x}}^{\mathrm{LD}}(g)$ decay to zero for large angles with different rates. $f_{\mathrm{x}}^{\mathrm{WK}}(g)$ decays with a Gaussian rate [equation (49)] and $f_{\mathrm{x}}^{\mathrm{LD}}(g)$ proportional to $1 / g^{4}$ [equation (57)]. On the other hand, $f_{\mathrm{x}}^{\mathrm{Kl}}(g)$ never decays to zero and instead approximates to a constant value [equation (53)].

Fig. 4 shows the calculated electron-density distribution versus the three-dimensional radius $r$ for $\operatorname{Ag}(Z=47)$. The calculated electron-density distribution at small radial distances from the atomic nucleus is shown in Fig. 4(a). $\rho^{\mathrm{WK}}(r)$, $\rho^{\mathrm{Kl}}(r)$ and $\rho^{\mathrm{LD}}(r)$ are positive functions that monotonically decay to zero in contrast to $\rho^{\mathrm{PRDW}}(r)$. Both $\rho^{\mathrm{PRDW}}(r)$ [equation (46)] and $\rho^{\mathrm{Kl}}(r)$ [equation (50)] present a singularity at $r=0$, which is forbidden for the electron-density distribution. $\rho^{\mathrm{LD}}(r)$ [equation (58)] is the only one that exhibits the correct asymptotic behaviour close to the nucleus, which is known to decay with an exponential rate (Weinstein et al., 1975; Sen \& Politzer, 1989). There is a difference by a factor of 15 between the calculated electron-density distribution at the atomic nucleus for $\rho^{\mathrm{WK}}(0)=18141.47 \AA^{-3}$ and $\rho^{\mathrm{LD}}(0)=$

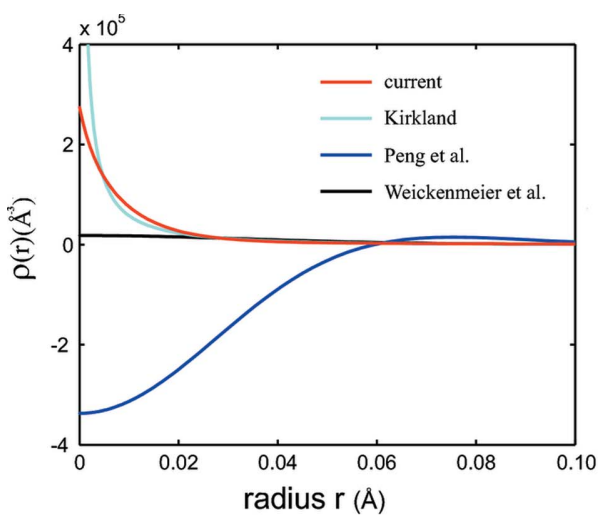

(a)

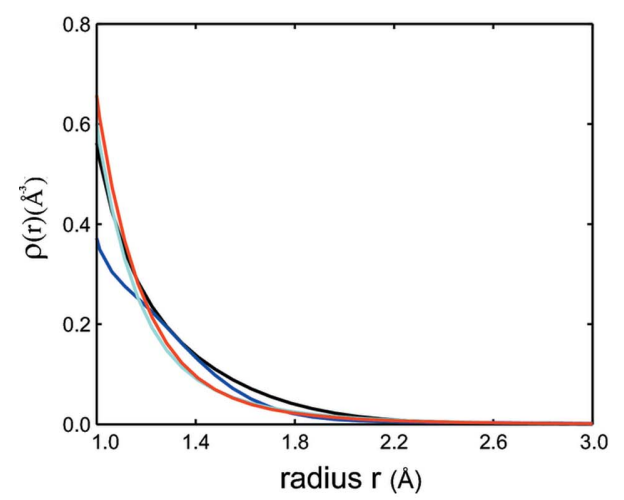

Figure 4

(b)

Comparison of different calculated electron charge distribution versus the three-dimensional radius $r$ for Ag. The electron charge distribution (a) at $r=0$ and $(b)$ at large distance from the atomic nucleus. 
$275435.86 \AA^{-3}$. This large difference arises from the poor quality of the fit of the Weickenmeier and Kohl parameterizations and the exclusive use of Gaussians as basis functions to express $\rho(r)$. Fig. 4(b) shows the electron-density distribution at large distances from the atomic nucleus. In this range, all the parameterizations of $\rho(r)$ have positive values; however, they display differences between them which decrease at large radial distances.

Fig. 5 shows the calculated atomic potential versus the three-dimensional radius $r$ for $\operatorname{Ag}(Z=47)$. Fig. 5(a) shows the calculated atomic potential at small radial distances from the atomic nucleus. $V^{\mathrm{WK}}(r), V^{\mathrm{Kl}}(r)$ and $V^{\mathrm{LD}}(r)$ exhibit the correct asymptotic behaviour close to the nucleus, which is known to be proportional to $1 / r$ [equations (51), (55) and (59)]. In contrast, $V^{\mathrm{PRDW}}(r)$ [equation (47)] shows a Gaussian behaviour. Fig. 5(b) shows the calculated atomic potential at large radial distances from the atomic nucleus. In this range, $V^{\mathrm{WK}}(r)$ differs from the other parameterizations, which show similar decaying behaviours.

These results show that our parameterized form of the electron scattering factor and its derived quantities such as $\mathrm{X}$-ray scattering factor, atomic potential and electron-density distribution is the only one that displays the correct behaviour in all ranges.

$\rho^{\mathrm{LD}}(r)$ parameterization can also be used in other fields to derive some relevant quantities. Table 2 shows the contact

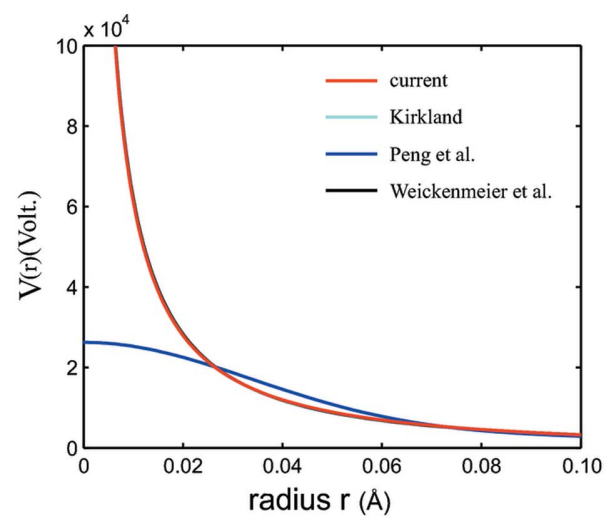

(a)

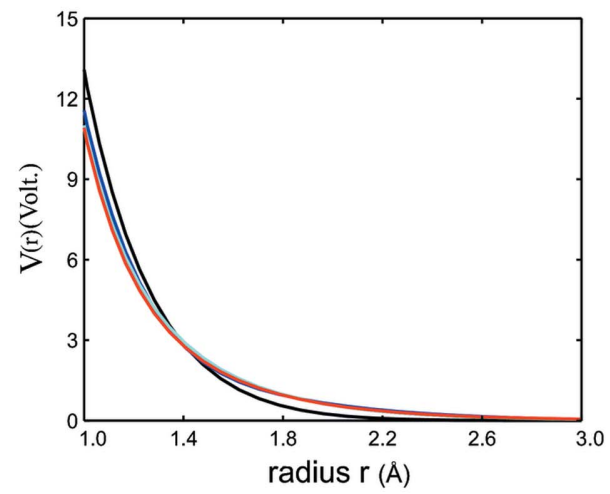

(b)

Figure 5

Comparison of different calculated atomic potentials versus the threedimensional radius $r$ for Ag. The atomic potentials $(a)$ at $r=0$ and $(b)$ at large distance from the atomic nucleus.
Table 2

Comparison of the electron-density distribution at the nucleus.

$\rho^{*}(0)$ values are taken from Angulo et al. (1990), Pacios (1991).

\begin{tabular}{rrrr}
\hline$Z$ & $\rho^{\mathrm{WK}}(0)$ & \multicolumn{1}{c}{$\rho^{\mathrm{LD}}(0)$} & \multicolumn{1}{c}{$\rho^{*}(0)$} \\
\hline 2 & 41.22 & 24.26 & 24.29 \\
3 & 59.91 & 92.92 & 93.36 \\
4 & 122.65 & 237.49 & 239.08 \\
5 & 269.62 & 491.77 & 485.78 \\
6 & 380.10 & 889.99 & 860.78 \\
7 & 600.41 & 1428.66 & 1391.07 \\
8 & 1236.69 & 2149.76 & 2105.31 \\
9 & 889.03 & 3084.49 & 3027.37 \\
10 & 1121.17 & 4244.11 & 4184.95 \\
11 & 1089.30 & 5759.08 & 5626.96 \\
12 & 4707.28 & 7514.44 & 7380.86 \\
13 & 2917.44 & 9645.94 & 9467.31 \\
14 & 6227.70 & 12092.59 & 11915.60 \\
15 & 2022.11 & 14985.69 & 14753.95 \\
\hline
\end{tabular}

density (which is the electron density at the position of the atomic nucleus) calculated from the parameterizations of $\rho^{\mathrm{WK}}(r)$ and $\rho^{\mathrm{LD}}(r)$, compared with the corresponding HartreeFock results.

In spite of the fact that $f_{\mathrm{e}}(\mathrm{g})$ was fitted using relativistic Hartree-Fock data (Kirkland, 1998), our parameterized $\rho(r)$ gives similar values to the numerical Hartree-Fock data of $\rho(0)$ (Angulo et al., 1990; Pacios, 1991). This is due to the fact that relativistic effects are small for low atomic numbers. On the other hand, $\rho^{\mathrm{WK}}(0)$ shows a large deviation from $\rho^{*}(0)$. This result validates our parameterization and allows its use in other fields.

Kato's cusp condition given in equation (22), which holds only for non-relativistic electron-density calculations, can be replaced for more rigorous constraints. For instance, the parameterized $f_{\mathrm{e}}(g)$ has to reproduce exactly the different moments of the electron distribution $\left\langle r^{k}\right\rangle$ defined as

$$
\left\langle r^{k}\right\rangle=\int r^{k} \rho(r) \mathrm{d} \mathbf{r}=4 \pi \int_{0}^{\infty} r^{k+2} \rho(r) \mathrm{d} r
$$

We want to point out that our fitting procedure has used two moments of the electron distribution $\left\langle r^{0}\right\rangle=Z$ and $\left\langle r^{2}\right\rangle=3 a_{0} f_{\mathrm{e}}(0)$ as constraints.

In principle, it would be better to parameterize the numerical radial atomic potential (which is related to the atomic charge distribution via Poisson's equation) rather than the electron scattering factors. Thus, this requires knowledge of the tabulated values of radial electron-density distribution for all the atoms.

\section{Conclusions}

We have developed a new algorithm and computer code to perform accurate parameterization of the numerical X-ray and electron scattering factors. The code was applied successfully to fit the tabulated electron scattering factors for all 103 neutral atoms for $g$ values in the range of $0<g<12 \AA^{-1}$. The new parameterized electron scattering factor was expanded by using the analytic non-relativistic 
hydrogen electron scattering factors as basis functions. The inclusion of the correct physical constraints in the electron scattering factor and its derived quantities such as the X-ray scattering factor, the electron-density distribution and the atomic potential allows us to use the new parameterization in different fields. In terms of quality of the fit $\sigma$, our proposed parameterization of the electron scattering factor is one order of magnitude better than the previous analytic fittings.

\section{APPENDIX $A$ \\ Parameterization of the electron scattering factor and its derived quantities}

In Smith \& Burge (1962), Cromer \& Waber (1965), Doyle \& Turner (1968) and Peng et al. (1996) the electron scattering factor and its derived quantities are parameterized as

$$
\begin{gathered}
f_{\mathrm{e}}(g)=\sum_{i=1}^{n_{g}} a_{i} \exp \left(-b_{i} g^{2}\right), \\
f_{\mathrm{x}}(g)=Z-\sum_{i=1}^{n_{g}} 2 \pi^{2} a_{0} a_{i} g^{2} \exp \left(-b_{i} g^{2}\right), \\
\rho(r)=Z \delta(r)+\sum_{i=1}^{n_{g}} \frac{\pi^{11 / 2} a_{0} a_{i}}{b_{i}^{7 / 2}}\left(2 r^{2}-\frac{3 b_{i}}{\pi^{2}}\right) \exp \left(-\frac{\pi^{2} r^{2}}{b_{i}}\right), \\
V(r)=\sum_{i=1}^{n_{g}} \frac{\pi^{3 / 2} a_{i}}{\kappa^{3 / 2}} \exp \left(-\frac{\pi^{2} r^{2}}{b_{i}}\right) .
\end{gathered}
$$

In Weickenmeier \& Kohl (1991) the electron scattering factor and its derived quantities are parameterized as

$$
\begin{gathered}
f_{\mathrm{e}}(g)=\sum_{i=1}^{n_{w}} a_{i} \frac{\left[1-\exp \left(-b_{i} g^{2}\right)\right]}{g^{2}}, \\
f_{\mathrm{x}}(g)=\sum_{i=1}^{n_{w}} 2 \pi^{2} a_{0} a_{i} \exp \left(-b_{i} g^{2}\right), \\
\rho(r)=\sum_{i=1}^{n_{w}} \frac{2 \pi^{7 / 2} a_{0} a_{i}}{b_{i}^{3 / 2}} \exp \left(-\frac{\pi^{2} r^{2}}{b_{i}}\right), \\
V(r)=\sum_{i=1}^{n_{w}} \frac{\pi \alpha_{i}}{\kappa} \frac{\operatorname{Erfc}\left(\frac{\pi r}{b_{i}}\right)}{r} .
\end{gathered}
$$

In Kirkland (1998) the electron scattering factor and its derived quantities are parameterized as

$$
\begin{gathered}
f_{\mathrm{e}}(g)=\sum_{i=1}^{n_{l}} \frac{a_{i}}{b_{i}+g^{2}}+\sum_{i=1}^{n_{g}} c_{i} \exp \left(-d_{i} g^{2}\right), \\
f_{\mathrm{x}}(g)=Z-\sum_{i=1}^{n_{l}} \frac{2 \pi^{2} a_{0} a_{i} g^{2}}{b_{i}+g^{2}}-\sum_{i=1}^{n_{g}} 2 \pi^{2} a_{0} c_{i} g^{2} \exp \left(-d_{i} g^{2}\right),
\end{gathered}
$$

$$
\begin{aligned}
\rho(r)= & \left(Z-\sum_{i=1}^{n_{l}} 2 \pi^{2} a_{0} a_{i}\right) \delta(r)+\sum_{i=1}^{n_{l}} 2 \pi^{3} a_{0} a_{i} b_{i} \frac{\exp \left(-2 \pi b_{i}^{1 / 2} r\right)}{r} \\
& +\sum_{i=1}^{n_{g}} \frac{\pi^{11 / 2} a_{0} c_{i}}{d_{i}^{7 / 2}}\left(2 r^{2}-\frac{3 d_{i}}{\pi^{2}}\right) \exp \left(-\frac{\pi^{2} r^{2}}{d_{i}}\right) \\
V(r) & =\sum_{i=1}^{n_{l}} \frac{\pi \alpha_{i}}{\kappa} \frac{\exp \left(-\frac{2 \pi r}{b_{i}^{1 / 2}}\right)}{r}+\sum_{i=1}^{n_{g}} \frac{\pi^{3 / 2} c_{i}}{\kappa d_{i}^{3 / 2}} \exp \left(-\frac{\pi^{2} r^{2}}{d_{i}}\right)
\end{aligned}
$$

For our proposed parameterization, the electron scattering factor and its derived quantities are expressed as

$$
\begin{gathered}
f_{\mathrm{e}}(g)=\sum_{i=1}^{n_{h}} \frac{a_{i}\left(2+b_{i} g^{2}\right)}{\left(1+b_{i} g^{2}\right)^{2}}, \\
f_{\mathrm{x}}(g)=\sum_{i=1}^{n_{h}} \frac{2 \pi^{2} a_{0} a_{i}}{b_{i}\left(1+b_{i} g^{2}\right)^{2}}, \\
\rho(r)=\sum_{i=1}^{n_{h}} \frac{2 \pi^{4} a_{0} a_{i}}{b_{i}^{5 / 2}} \exp \left(-\frac{2 \pi r}{b_{i}^{1 / 2}}\right), \\
V(r)=\sum_{i=1}^{n_{h}} \frac{\pi^{2} a_{i}}{\kappa b_{i}^{3 / 2}}\left(\frac{b_{i}^{1 / 2}}{\pi r}+1\right) \exp \left(-\frac{2 \pi r}{b_{i}^{1 / 2}}\right) .
\end{gathered}
$$

\section{References}

Angulo, J. C., Dehesa, J. S. \& Galvez, F. J. (1990). Phys. Rev. A, 42, 641-644.

Bethe, H. (1928). Ann. Phys. 392, 55-129.

Bethe, H. (1930). Ann. Phys. 5, 325-400.

Corona, A., Marchesi, M., Martini, C. \& Ridella, S. (1987). ACM Trans. Math. Softw. 13, 262-280.

Coulthard, M. A. (1967). Proc. Phys. Soc. 91, 44-49.

Cowley, J. M. \& Moodie, A. F. (1957). Acta Cryst. 10, 609-619.

Cromer, D. T. \& Waber, J. T. (1965). Acta Cryst. 18, 104-109.

Desclaux, J. P. (1975). Comput. Phys. Commun. 9, 31-45.

Doyle, P. A. \& Turner, P. S. (1968). Acta Cryst. A24, 390-397.

Dyall, K. G., Grant, I. P., Johnson, C. T., Parpia, F. A. \& Plummer, E. P. (1989). Comput. Phys. Commun. 55, 425-456.

Fox, A. G., O'Keefe, M. A. \& Tabbernor, M. A. (1989). Acta Cryst. A45, 786-793.

Goodman, P. \& Moodie, A. F. (1974). Acta Cryst. A30, 280-290.

Grant, I. P. (1961). Proc. R. Soc. London Ser. A, 262, 555-576.

Grant, I. P. (1970). Adv. Phys. 19, 747-811.

Grant, I. P., McKenzie, B. J., Norrington, P. H., Mayer, D. F. \& Pyper, N. C. (1980). Comput. Phys. Commun. 21, 207-231.

Howie, A. \& Whelan, M. J. (1961). Proc. R. Soc. London Ser. A, 263, 217-237.

Ibers, J. A. (1958). Acta Cryst. 11, 178-183.

Ingber, L. (1993). Math. Comput. Model. 18, 29-57.

Ishizuka, K. \& Uyeda, N. (1977). Acta Cryst. A33, 740-749.

Kato, T. (1957). Commun. Pure Appl. Math. 10, 151-177.

Kirkland, E. J. (1998). Advanced Computing in Electron Microscopy. New York: Springer.

Kirkpatrick, S., Gelatt, C. D. Jr \& Vecchi, M. P. (1983). Science, 220, 671-680.

Lobato, I. \& Van Dyck, D. (2012). Ultramicroscopy, 119, 63-71.

Mastalerz, R., Widmark, P., Roos, B. O., Lindh, R. \& Reiher, M. (2010). J. Chem. Phys. 133, 144111. 
Matsumoto, M. \& Nishimura, T. (1998). ACM Trans. Model. Comput. Simul. 8, 3-30.

Metropolis, N., Rosenbluth, A. W., Rosenbluth, M. N., Teller, A. H. \& Teller, E. (1953). J. Chem. Phys. 21, 1087-1092.

Mott, N. F. (1930). Proc. R. Soc. London Ser. A, 127, 658-665.

Pacios, L. F. (1991). J. Phys. Chem. 95, 10653-10658.

Peng, L.-M., Ren, G., Dudarev, S. L. \& Whelan, M. J. (1996). Acta Cryst. A52, 257-276.

Press, W. H., Teukolsky, J. A., Vetterling, W. T. \& Flannery, B. P. (2007). Numerical Recipes, 3rd ed. Cambridge University Press.

Rez, D., Rez, P. \& Grant, I. (1994). Acta Cryst. A50, 481-497.
Sen, K. D. \& Politzer, P. (1989). J. Chem. Phys. 38, 4370-4372.

Smith, G. H. \& Burge, R. E. (1962). Acta Cryst. 15, 182-186.

Sperber, G. (1971). Int. J. Quantum Chem. 5, 189-214.

Steiner, E. (1963). J. Chem. Phys. 39, 2365-2366.

Van Dyck, D. (1979). Phys. Status Solidi A, 52, 283-292.

Van Dyck, D. (1980). J. Microsc. 119, 141-152.

Van Dyck, D. \& Coene, W. (1984). Ultramicroscopy, 15, 29-40.

Wang, W.-P. \& Parr, R. G. (1977). Phys. Rev. A, 16, 891-902.

Weickenmeier, A. \& Kohl, H. (1991). Acta Cryst. A47, 590-597.

Weinstein, H., Politzer, P. \& Srebrenik, S. (1975). Theor. Chim Acta, 38, 159-163. 